\title{
THREE-DIMENSIONAL RECONSTRUCTION OF CHROMOSOME PAIRING DURING THE ZYGOTENE STAGE OF MEIOSIS IN LILIUM LONGIFLORUM (THUNB.)
}

by

\author{
PREBEN BACH HOLM \\ Department of Physiology - Carlsberg Laboratory \\ Gamle Carlsberg Vej 10 - DK-2500 Copenhagen, Valby
}

Key words: electron microscopy, reconstruction, early zygotene

Lilium longiflorum, synaptinemal complex

\begin{abstract}
Chromosome pairing in early zygotene in Lilium longiflorum has been studied by three-dimensional reconstructions at the electron microscopical level. One nucleus has been completely reconstructed, and the pairing behaviour and the length of the 12 partially synapsed bivalents was analysed and measured. The total length of the 24 chromosomes comprises $7,4 \mathrm{~mm}$, and pairing is initiated at several sites along the chromosomes. Analysis of the pattern and temporal sequence of pairing suggests that all regions of the lateral component contain the required specificity for site-to-site matching. Measurements of the length of the unpaired homologous regions demonstrate that identical chromosome length is secured for both homologues before pairing is initiated. Pairing is accomplished by the attachment of a piece of central region to one of the lateral components after rotation of the associated chromatin, followed by subsequent binding of the central region to the homologous lateral component. The majority of the telomeres are attached via a special substance to a restricted portion of the nuclear envelope. The occurrence of chromatin associated structures at the nuclear envelope in combination with the highly polarized distribution of nuclear pores suggests that considerable membrane activity of possible significance for chromosome movements takes place during this interval. The observations are discussed in relation to current hypotheses on the structure and biochemistry of early meiotic chromosomes and the processes of alignment and pairing.
\end{abstract}

\section{INTRODUCTION}

The elucidation of the genetical, biochemical and structural mechanisms underlying pairing of homologous chromosomes during the zygotene stage of eukaryotic meiosis is of profound significance for the understanding of meiotic crossing-over. An increasing amount of ultrastructural evidence suggests that the synaptinemal complex at pachytene provides the structural framework for specific site-to-site matching and crossing-over $(20,41,62)$. Likewise, bio- chemical evidence in favour of precise pairing during zygotene as well as recombinational events by DNA break-repair mechanisms in pachytene is accumulating (54).

The three-dimensional reconstruction of synaptinemal complexes has recently confirmed and extended the classical cytogenetic interpretations of chromosome behaviour during meiosis, but only few reconstructions have been conducted on the zygotene stage. The nature and temporal sequence of chromosome pairing as 
well as the overall organization of the leptotene and early zygotene chromosomes are still not known in detail, and the biochemical characteristics of meiotic prophase have not yet been correlated in detail with the ultrastructural observations.

In the analysis of such questions, threedimensional reconstructions of the stage of $z y-$ gotene in Lilium longiflorum are relevant. In a series of experiments HOTTA, STERN and coworkers have investigated the biochemistry of chromosome pairing, demonstrating that precise pairing might be accomplished by DNA-DNA matching of a minor fraction of unique DNA-sequences unreplicated during the preceding S-phase. This primary pairing is followed by replication of the DNA regions and the homologous chromosome regions are assumed to be stabilized via the central region of the synaptinemal complex. Several proteins of relevance to this primary matching process have been identified and studied (54). Moreover, the behaviour of the Lilium longiflorum genome during premeiotic interphase and leptotene as well as during the somatic cell cycle has been analysed at the light microscopical level $(22,23,55)$.

The present paper is addressed to the structural aspects of early zygotene chromosome pairing in Lilium longiflorum. Reconstructions and length measurements of the 12 partially synapsed bivalents are presented, as well as structural details of the nucleus and the processes of pairing. Moreover, it has been the aim of the present study to obtain quantitative and qualitative information on nuclear structure for further investigations of the presynaptic stages in Lilium longiflorum.

\section{MATERIALS AND METHODS}

\subsection{Plant material}

Flower buds were obtained from a clone of Lilium longiflorum grown in the Royal College of Forestry phytotron in Stockholm. This particular clone has also provided material for in- vestigations of the presynaptic as well as the somatic stages $(22,23,55)$ and its growth characteristics during early meiosis have been dealt with in great detail. The original observation by ERICKSON (12) of a correlation between bud length, anther length and stage of meiocytes in the anthers has only been confirmed to some extent for the short presynaptic stages. Bud length however, does provide a general guide for isolation of the stages thereby facilitating the study of the temporal sequence of meiotic events.

\subsection{Electron microscopy}

Flower buds in the appropriate stages of development were excised from the plants and their length measured. After removal of the perianth, each anther was measured separately on millimeter paper, slit longitudinally through the connective and the anther halves transferred to fixation vials containing $4 \%$ formaldehyde in $0,05 \mathrm{M}$ potassium phosphate buffer, $\mathrm{pH} \mathrm{7,2.} \mathrm{After} \mathrm{one} \mathrm{hour} \mathrm{each} \mathrm{anther} \mathrm{haif} \mathrm{was}$ divided into three equally large parts (tip, mid and base) and fixed for an additional 30 minutes in formaldehyde. An equal volume of $4 \%$ glutaraldehyde in the same buffer was added and the material fixed for a further $1 \frac{1}{2}$ hours. Formaldehyde was prepared as a $40 \%$ stock solution by alkaline hydrolysis of paraformaldehyde at $70^{\circ} \mathrm{C}$ and the $\mathrm{pH}$ adjusted to 7.0. Commercially available glutaraldehyde was purified by repeated treatments with charcoal and its concentration and purity measured as described by ANDERSON (1). Samples with a purity index below 1 were utilized as stock solutions for fixation.

After several buffer washes postfixation was carried out for $21 / 2$ hours at room temperature in $2 \% \mathrm{OsO}_{4}$ in $0,05 \mathrm{M}$ potassium phosphate buffer, $\mathrm{pH} 7,2$. The material was subsequently washed several times in buffer and distilled water and dehydrated through a graded ethanol-water series. After a final one hour immersion in propylene oxide, the material was infiltrated with SPURR's low-viscosity epoxy resin

Abbreviations: NOR - nucleolus organizing region, $\mathrm{K}_{\mathrm{NOR}}$ - dense chromatic knob of nucleolus organizer region, $\mathrm{L}_{\mathrm{NOR}}$ - light staining chromatin of nucleolus organizer region. 


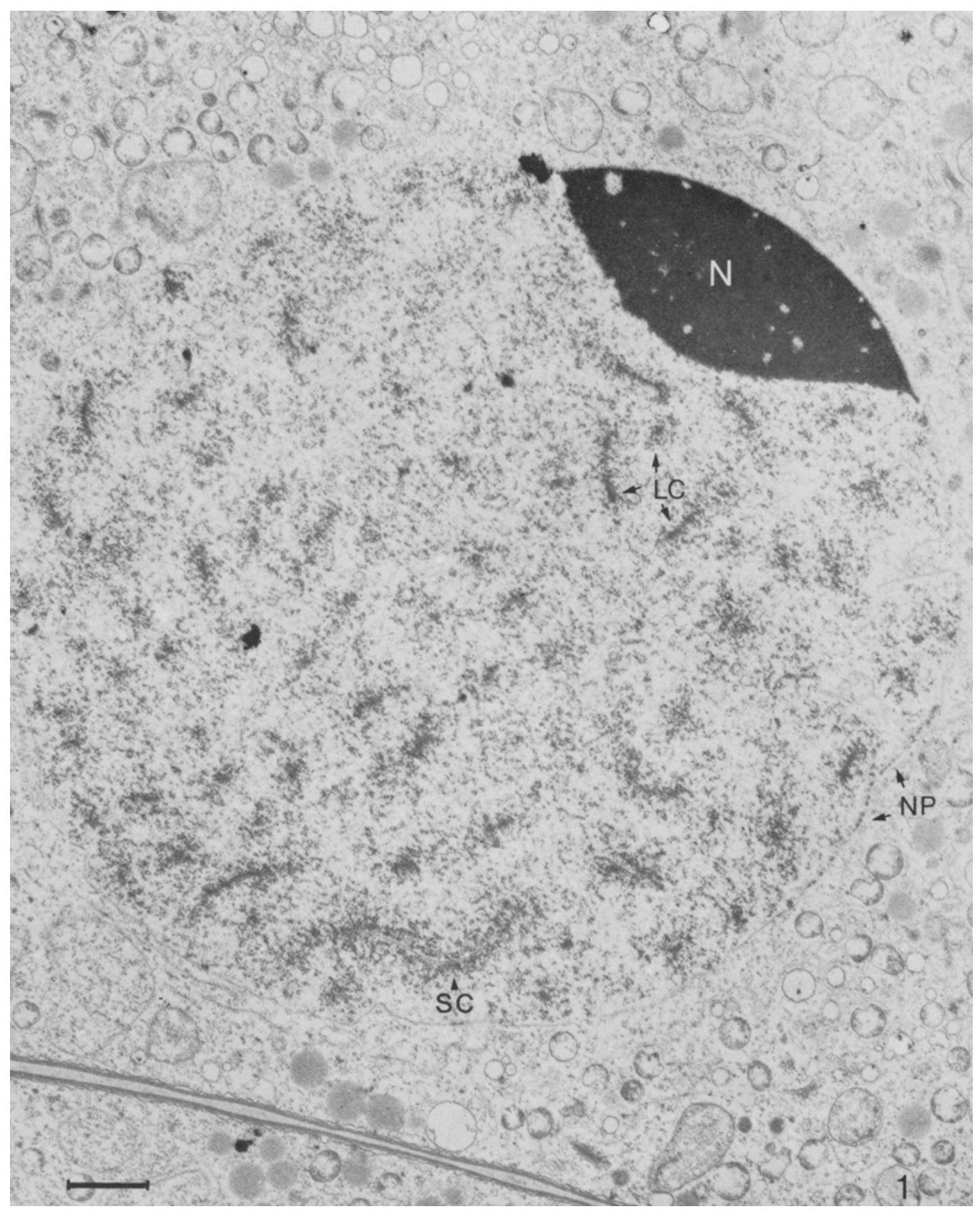

Figure 1: Survey micrograph of an early zygotene nucleus. The nucleolus is flattened along the nuclear envelope. A minor region of aggregated nuclear pores is evident. In the nucleus several unpaired and paired chromosome regions can be observed. SC, synaptinemal complex. LC, lateral component. N, nucleolus. NP, nuclear pores. $x 5.500(\mathrm{~B} \mathrm{ar}=2,0 \mu \mathrm{m})$

(53), left overnight in pure resin and the next day transferred to fresh resin for flat embedding in plastic trays. Polymerization was carried out at $70^{\circ} \mathrm{C}$ for 24 hours. 
All material was analysed for stage determination and fixation by phase contrast microscopy of thick sections. Selected material was subjected to ultrathin sectioning using a Reichert Om U3 ultramicrotome equipped with a diamond knife. An appropriate number of serial sections were collected and removed from the knife trough in a water drop in the hole of a single slot grid. After mounting in a micromanipulator this grid was oriented with respect to a formvar-covered, carbon-coated single slot grid, gently lowered and when touching, the sections were transferred by sucking away the water. Series comprising approximately 600 consecutive sections were stained for $30 \mathrm{~min}$ utes at $40^{\circ} \mathrm{C}$ in saturated uranyl acetate in $50 \%$ ethanol in water, washed several times in water and poststained for 15 minutes at room temperature in lead citrate. The staining was performed in a Hiraoka staining kit.

The sections were analysed in a Siemens 102 electron microscope at $80 \mathrm{kV}$. Micrographs for reconstruction purposes were taken at a negative magnification of $x 2000$, and printed to a total magnification of $\times 8000$.

\subsection{Reconstruction}

Reconstructions were carried out as described by GILLIES (16). Chromosomes and other nuclear structures were traced from micrographs of ten sections onto transparent plastic sheets, and by stacking the sheets a three-dimensional model of the nucleus emerged. Chromosome lengths were calculated for every ten sections using the Pythagorean Theorem: $a=\sqrt{b^{2}+c^{2}}$ (a: corrected chromosome length, b: $10 \times$ section thickness, c: projected chromosome length). Section thickness was calculated by dividing the diameter of the nucleus with the number of sections required to encompass the whole nucleus (nuclear diameter: $31,3 \mu \mathrm{m}$, num- ber of sections: 390 , section thickness: $800 \AA$ ).

In the present study two serially sectioned nuclei were photographed and one of them reconstructed completely.

\section{RESULTS}

\subsection{General comments}

For the analysis of early meiotic events the occurrence of a basipetal developmental gradient within the anther is especially favourable (23), and anthers containing meiocytes at late leptotene in the base and at early zygotene in the tip are regularly found. Besides chromosome pairing, which can be observed on both squashed and sectioned material, other traits characterize the transition from late leptotene to early zygotene. The cells acquire a more spherical shape, whereby the intercellular volume is increased. The structure and behaviour of the nucleoli aids in the identification of the stages. At late leptotene the nucleoli are flattened onto the nuclear envelope and fuse. Serial sectioning and reconstructions of 65 nuclei at the light microscopical level have demonstrated that $50 \%$ of the early zygotene nuclei possess one nucleolus and the remaining nuclei two nucleoli generally in close proximity to each other. Cytomixis consisting of fragmentation of nuclei and migration of the fragments through cytoplasmic channels in the walls is encountered frequently at early zygotene as a result of fixation accidents and less frequently at leptotene or late zygotene. Ultrastructural work in progress supports the developmental sequence defined by light microscopy. Nuclei classified as late leptotene possess continuous lateral components, and in the one serially sectioned nucleus no synaptinemal complexes have been identified.

Figure 2: High magnification micrograph of unpaired and paired chromosomes. The unpaired chromosome shown in Figure 2a demonstrates the centrally located lateral component associated with the chromatin. Longitudinal and transverse sections of the synaptinemal complex are shown in Figures $2 \mathrm{~b}$ and $2 \mathrm{c}$. The lateral position of the lateral component is evident. SC, synaptinemal complex. LC, lateral component. $\mathrm{CH}$, chromatin. $\mathrm{CC}$, central component. $\mathrm{CR}$, central region.

$2 \mathrm{a} \times 80.000(\mathrm{Bar}=0,1 \mu \mathrm{m})$

$2 \mathrm{~b}-2 \mathrm{c} \times 120.000(\mathrm{Bar}=0,1 \mu \mathrm{m})$ 


\section{P. B. HoLm: Chromosome pairing in Lilium}
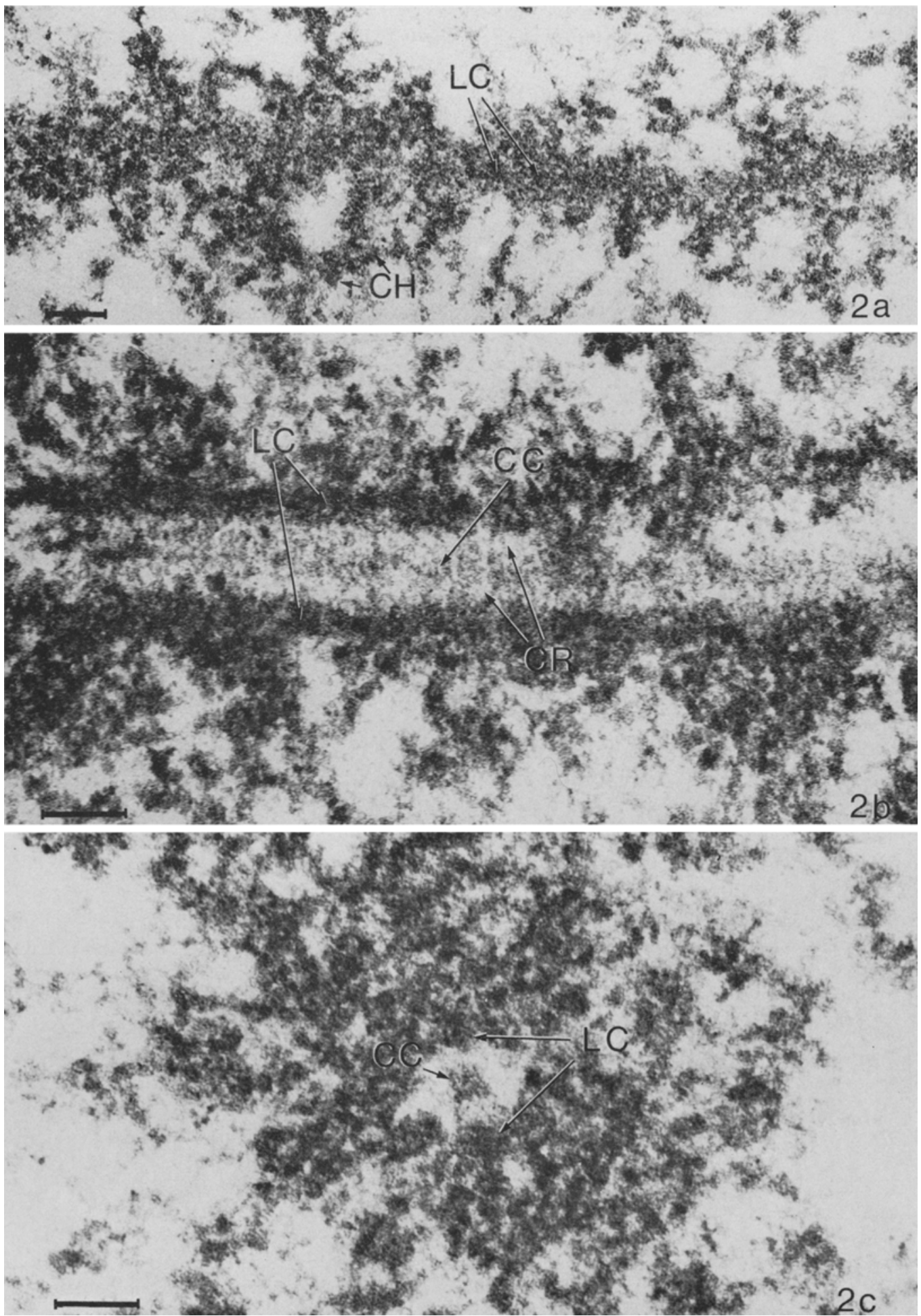


\subsection{Ultrastructure of zygotene nuclei}

3.2.1. Synaptinemal complex, lateral components, chromatin

The overall ultrastructure of early zygotene nuclei as observed at low magnification is shown in Figure 1. The most prominent feature is the occurrence of single unpaired chromosomes consisting of a lateral component, $300-400 \AA$ in diameter surrounded by chromatin organized in a loop-like fashion. The two chromatids cannot be recognized as separate units. The lateral components are often difficult to identify, being of approximately the same density as the surrounding chromatin after conventional fixation and staining. High magnification studies demonstrate a fine filamentous substructure of the lateral component (Fig. 2a) with connections to the surrounding chromatin and it is often possible to observe some degree of regularity either in the form of longitudinal or transverse fiber symmetry. Various degrees of compactness characterizes the associated chromatin with its beaded nucleosome structure. Closely grouped nucleosomes probably correspond to chromomeres.

Paired regions are less frequently observed. The synaptinemal complex reveals its typical tripartite organization of the two lateral components and the central region, the latter with a more distinct filamentous medial part, the central component. The diameter of the central region and central component are measured to be $1000 \AA$ and $300 \AA$ respectively. An elaborate system of fine filaments connects the lateral components with the central component which actually may be visible through a higher degree of compaction of the fibers. High magnification studies have occasionally demonstrated a thickening of the central region into a node-like structure (Fig. 9d). Similar local modifications of the central region have previously been de- scribed from Neurospora (16), Lilium longiflorum (37), various fungi (62) and Drosophila (6).

\subsubsection{Centromeres, knobs, nucleolus organizers (NOR)}

The recognition of centromeres in plant chromosomes at the ultrastructural level has been made possible after their identification through karyotype reconstruction in maize (17). With conventional fixation and contrasting methods centromeres in lily are less electron dense than the other chromatin regions and have a filamentous substructure largely devoid of the beaded nucleosomes (Figs. 3a, 3b). Lateral components and synaptinemal complexes pass uninterrupted through the centromere. In a few cases the centromeres have been observed to be bordered by distinct chromomeres (Fig. 3a). Electron dense loops extend from the lateral component within the centromere (Fig. 3b).

Knobs have an ultrastructure which is similar to that of centromeres (17). In lily they are a regular feature of the three nucleolus organizer regions (NOR). This chromosome region consists of a dense knob $\left(\mathrm{K}_{\mathrm{NOR}}\right)$ and an adjacent knob of lighter density ( $\mathrm{L}_{\mathrm{NOR}}$ ), which is partly enclosed in the nucleolus (Figs. 4a, 4b). This organization of the NOR region is identical to that described by GILLIES for Zea mays pachytene chromosomes (17). Lateral to the NOR a sphere of very fine filaments of low density (diffuse body Fig. 4a) is regularly found at early zygotene in Lilium longiflorum. Diffuse bodies have been identified in serially sectioned nuclei as far back as the premeiotic $G_{1}$ stage, occurring in extensive amounts either free in the nucleoplasm or associated with chromatin or with the NOR. In late leptotene most of them have disappeared except for those observed in asso-

Figure 3: Ultrastructure of the centromere region during early zygotene. In Figure 3a the synaptinemal complex is found in the middle of the centromere. The arrow denotes a region where the central region is associated with only one of the lateral components. (cf. Figure 9 and text). The centromere is on one side bordered by distinct chromomeres. In Figure $3 \mathrm{~b}$ arrows point to loops originating from the lateral component. $\mathrm{CE}$, centromere. $\mathrm{CH}$, chromatin. CRO, chromomere.

$3 \mathrm{a} \times 64.000(\mathrm{Bar}=0,1 \mu \mathrm{m})$

$3 \mathrm{~b} \times 100.000($ Bar $\times 0,1 \mu \mathrm{m})$ 


\section{P. B. HoLM: Chromosome pairing in Lilium}
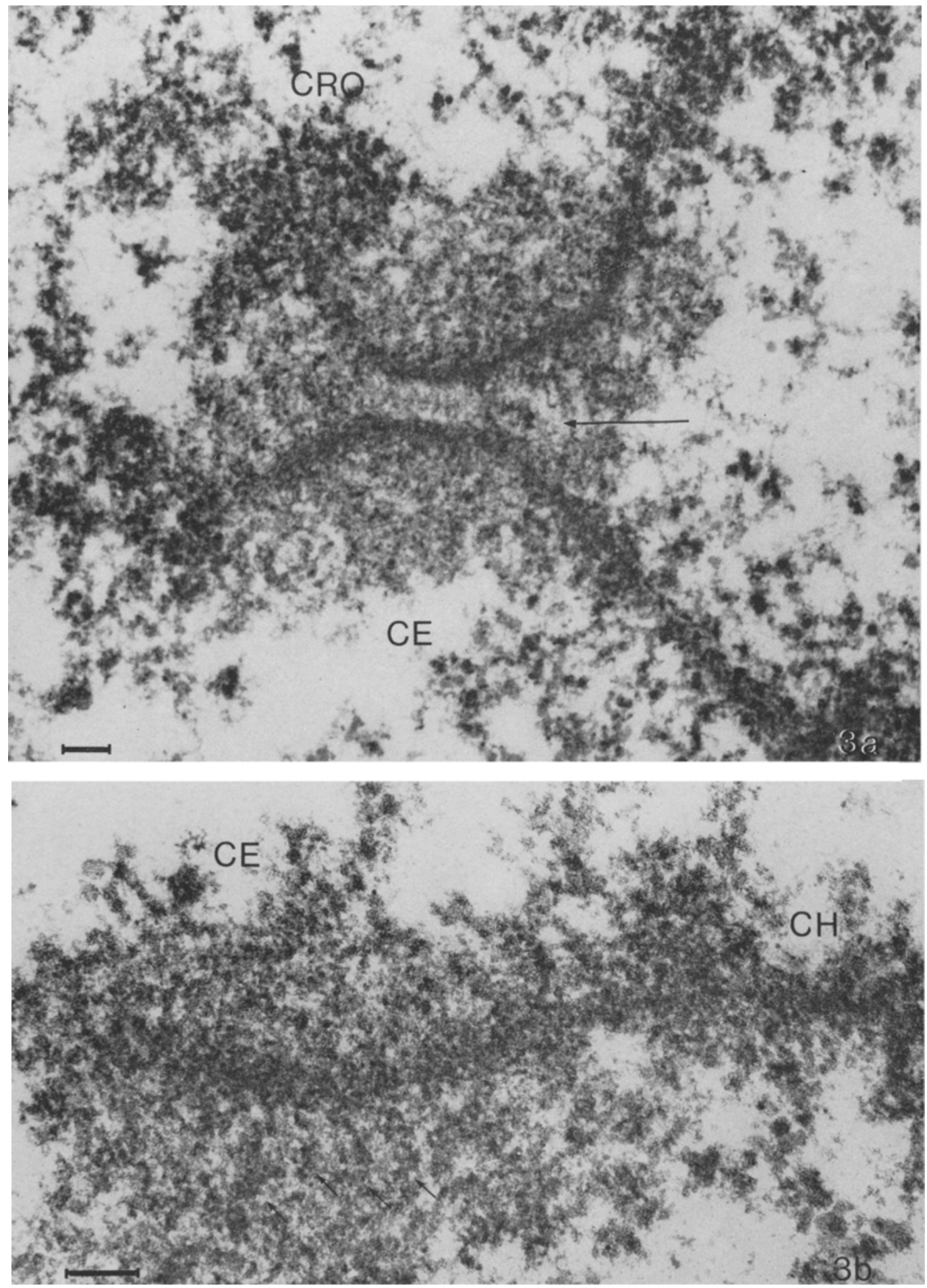


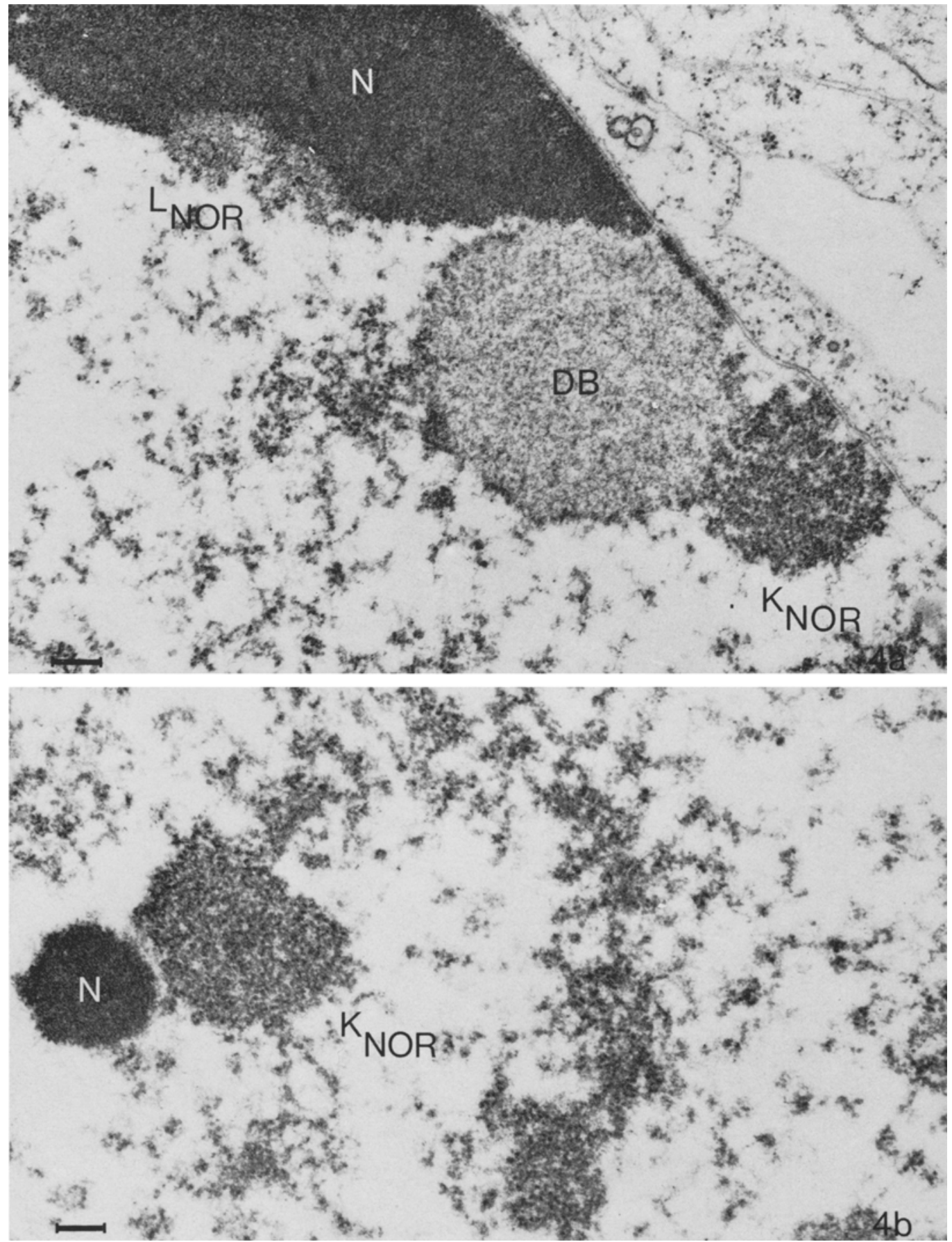

Figure 4: Ultrastructure of the nucleolus organizer region (NOR). In Figure $4 \mathrm{a}$ is shown the $\mathrm{L}_{\mathrm{NOR}}$ of chromosome 4 and the $K_{\text {NOR }}$ of chromosome 7. For explanation of terms, see text. Lateral to the NOR a sphere of low density filaments can be observed (diffuse body). Figure $4 \mathrm{~b}$ demonstrates the detached NOR of bivalent 3 . The $\mathrm{L}_{\text {NOR }}$ is localized below the $\mathrm{K}_{\mathrm{NOR}}$. A minor nucleolus is evident. $\mathrm{N}$, nucleolus. $\mathrm{DB}$, diffuse body. $4 \mathrm{a}-\mathrm{b} \times 32.000(\mathrm{Bar}=0,2 \mu \mathrm{m})$. 
ciation with the NORs. The diffuse bodies are accordingly not an integral part of the nucleolus organizer region. Chromosomes 2 and 3 were found homozygous for one small knob and chromosomes 3 and 7 heterozygous for two and one knobs respectively.

\subsubsection{Nuclear polarity, telomeres and the nuclear envelope}

The zygotene nucleus exhibits a highly polarized organization (Figs. 5,6). Of a total of 48 telomeres, 34 are attached to the nuclear envelope within a region comprising one-eighth of the total nuclear surface, 4 are localized in an adjacent area and the remaining 10 are free in the nucleoplasm. The major region of attachment is positioned lateral to the two nucleoli and close to two very distinct areas of aggregated nuclear pores. More precisely, the telomeres were attached to the smaller of the two nuclear pore fields and to the area between the two fields. The telomeres possess a special morphology. If bound to the inner membrane of the nuclear envelope a cone-shaped structure, consisting of fine fibers with a low density and devoid of nucleosomes is present (Fig. 7). This terminal structure in a spherical form is particularly evident for the free telomeres of bivalent (Fig. 7c) or univalent chromosomes. All free telomeres have been found to be associated with this structure when analysed at high magnification. This structure may be responsible for the attachment of telomeres to the envelope, the lateral components being separated through it from the inner membrane by a few hundred $\dot{A}$. Both the inner and the outer membrane have an increased electron density at the attachment sites, and the perilacunar space is bridged by fine fibers often extending a few hundred $\dot{A}$ into the cytoplasm (Fig. 7b). Direct association with microtubules has only been observed for one set of paired telomeres. Interstitial chromatin is also frequently bound to the inner membrane of the nuclear envelope with fine fibrillar material devoid of nucleosomes (Figs. 7d, e arrows). In other cases of chromatin association with the nuclear envelope the material was not observed.

The nuclear pores posses a distinct eightfold symmetry and a central plug or granule (Fig. 8).
The peripheral subunits of the pore taper off into filaments, often associated with chromatin (Figs. 7e, 8a, b). A similar organization of the subunits is evident on the cytoplasmic side, but filaments are fewer. Outside of the major and minor field of aggregated pores (Figs. 5 and 6) several small aggregates can be recognized, the rest of the nuclear envelope being virtually devoid of pores. Invaginations of the nuclear envelope, especially in the regions without pores, are evident.

To the inner membrane are attached at various places prominent roughly spherical plates, $500-600 \dot{A}$ in thickness and with an electron density similar to that of nucleolar material (Figs. 5, 6 and 8b). These structures are also abundant in mid leptotene. Chromatin has been observed to associate with this structure, but no direct connections between the plates and the components of the synaptinemal complex have been found.

\subsubsection{Ultrastructure of chromosome pairing}

Selected parts of the reconstructed zygotene chromosomes have been analysed at primary magnifications of 10-50.000 times, with special consideration of the junction between paired and unpaired regions. Due to the twisted nature of the chromosomes such regions are seldom included in a single section, necessitating reconstructions from a series of sections. The two unpaired homologous chromosomes diverge at various angles from the region of completed synaptinemal complex (Figs. 3a and 9). Continuity of the lateral components is observed but in the univalent portions of the pairing chromosomes they are located in the middle of the chromatin. In Fig. 9d a piece of the central region extends out from a well defined synaptinemal complex and is associated with the lateral component of only one of the diverging chromosomes. At this point its associated chromatin has rotated with respect to the lateral component, while in the homologue the rotation has not yet taken place. In the latter a piece of what is believed to be amorphous central region material is attached to the lateral component a little further away (Figs. 9e and $\mathrm{f}$ ). The attachment seems to initiate the rotation of the chromatin. Attachment of central region to one 


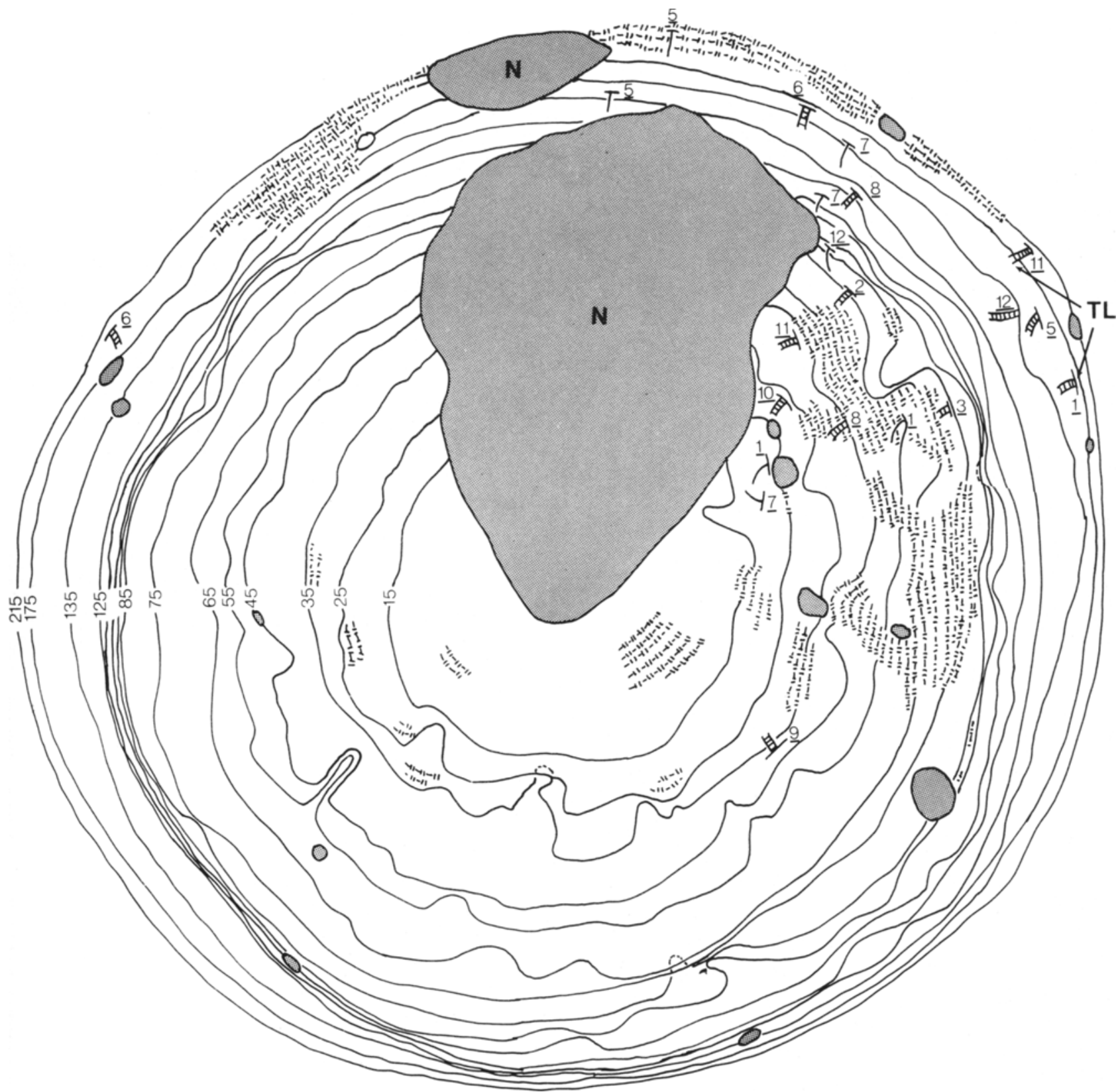

Figures 5 and 6: Reconstructions of nuclear envelope associated structures in the early zygotene nucleus. The top half of the nucleus is shown in Figure 5, the bottom half in Figure 6. The distribution of nucleoli, pores and dense plates is shown. Membrane associated telomeres are indicated and found in a limited area of the nuclear envelope. Deep invaginations of the nuclear envelope can be noted. N, nucleolus. TL, telomere. DP, dense plate. NP, nuclear pores. Numbers indicate section in which profile was found, underlined numbers designate chromosomes. 


\section{P. B. HoLm: Chromosome pairing in Lilium}

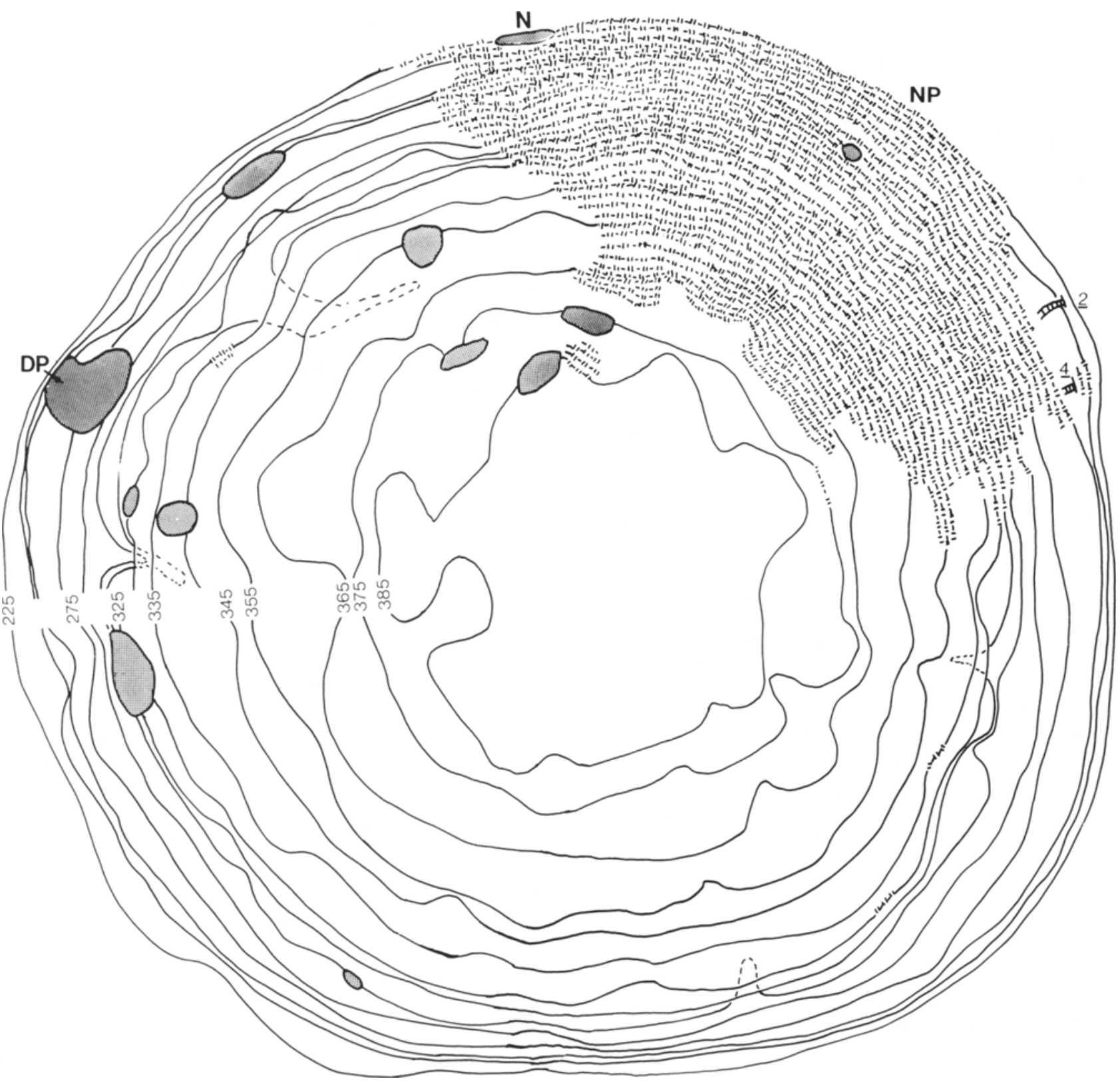



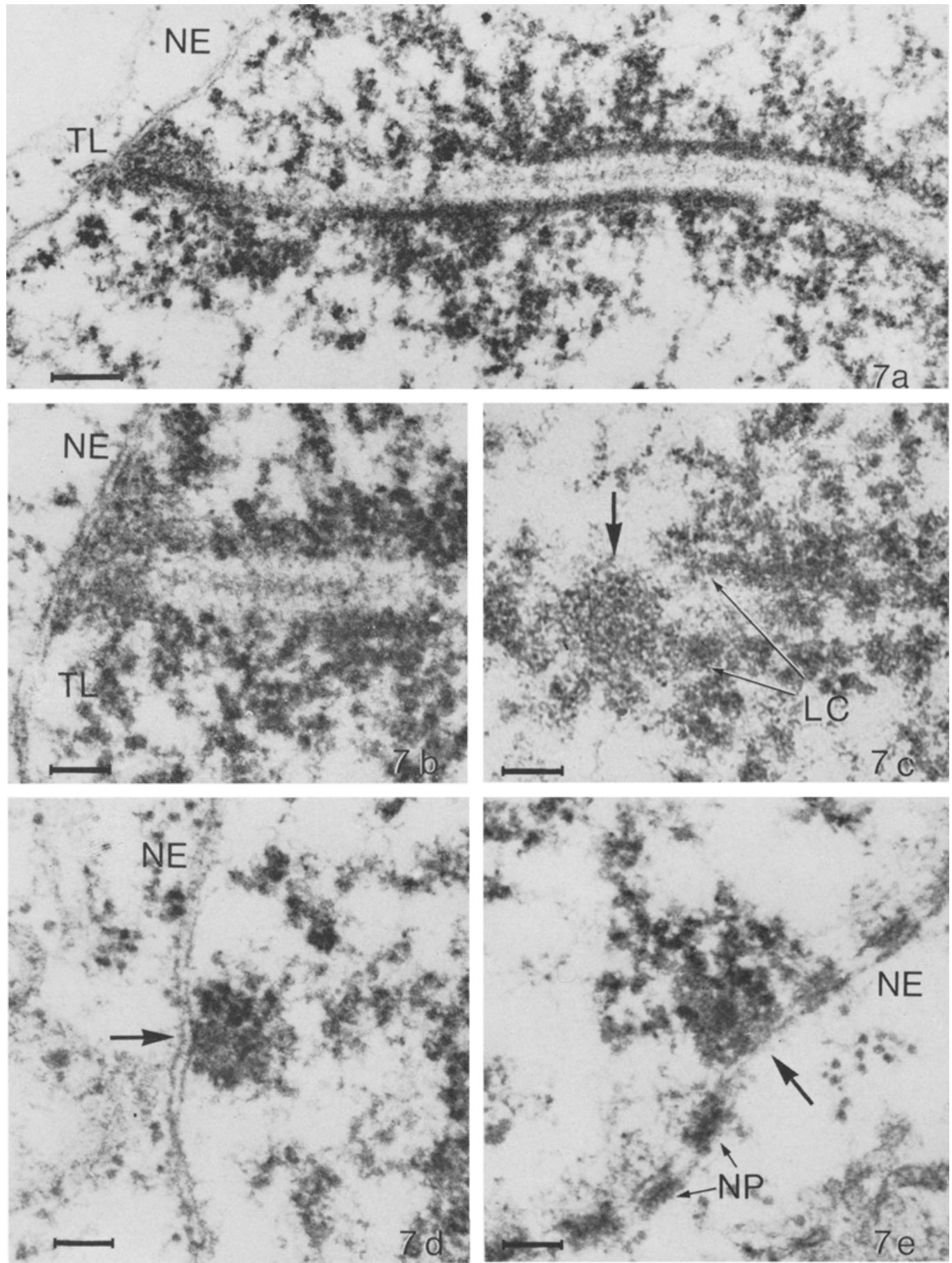

Figure 7: Attachment of telomeres and internal chromosome regions to the nuclear envelope. The binding of telomeres to the envelope via a substance differing from chromatin in having lower density and absence of nucleosomes is documented in Figures $7 \mathrm{a}$ and $7 \mathrm{~b}$. Fine filaments traversing the perilacunar space can be observed in Figure 7b. In Figure 7c is shown the end of the short arm of bivalent 4 located free in the nucleoplasm. The telomeres are paired with a synaptinemal complex, one lateral component of which is associated with a sphere of material (arrow) resembling that found at the binding site of telomeres to the nuclear envelope. In Figures $7 \mathrm{~d}$ and $7 \mathrm{e}$ is shown the binding of interstitial chromatin to the nuclear envelope via material of lower density than chromatin and with resemblance to the telomere associated material. (Indicated by thick arrows.) NE, nuclear envelope. LC, lateral components. TL, telomeres. NP, nuclear pores.

$7 \mathrm{a} \times 48.000(\mathrm{Bar}=0,2 \mu \mathrm{m}), 7 \mathrm{~b}-7 \mathrm{e} \times 80.000(\mathrm{Bar}=0,1 \mu \mathrm{m})$ 


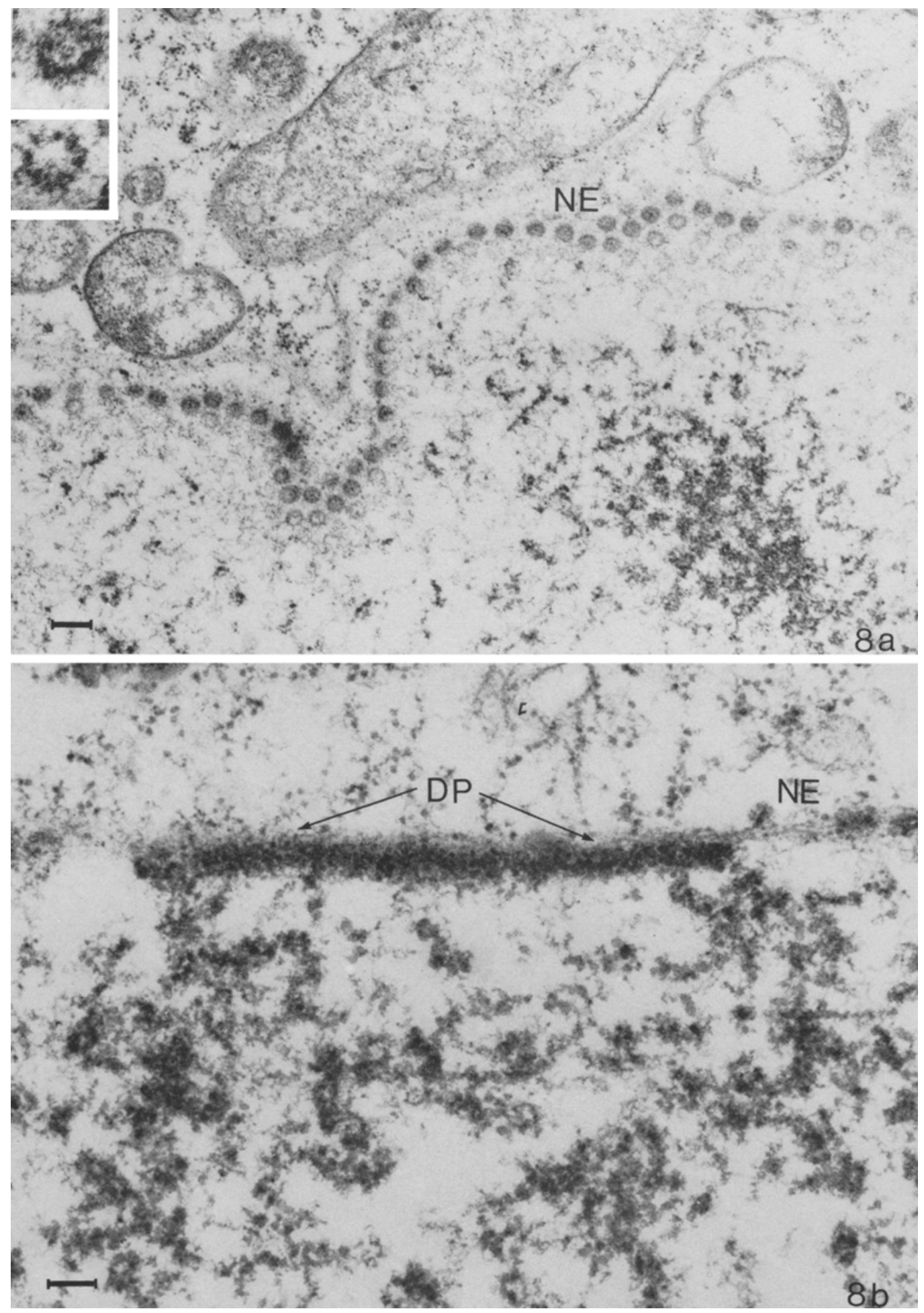

Figure 8: Aggregated nuclear pores and dense plate. Pores in the nuclear envelope are shown in Figure 8a, with inserts demonstrating the eight fold symmetry and the central plug of the nuclear pores. Filamentous projections from the peripheral subunits of the pores are evident. A dense plate is shown in Figure 8b. Chromatin can be observed to be connected to the material of the plate. NE, nuclear envelope. DP, dense plate.

$8 \mathrm{a} \times 25.700(\mathrm{Bar}=0,2 \mu \mathrm{m})$, inserts $\times 100.000$

$8 \mathrm{~b} \times 64.000(\mathrm{Bar}=0,2 \mu \mathrm{m})$. 

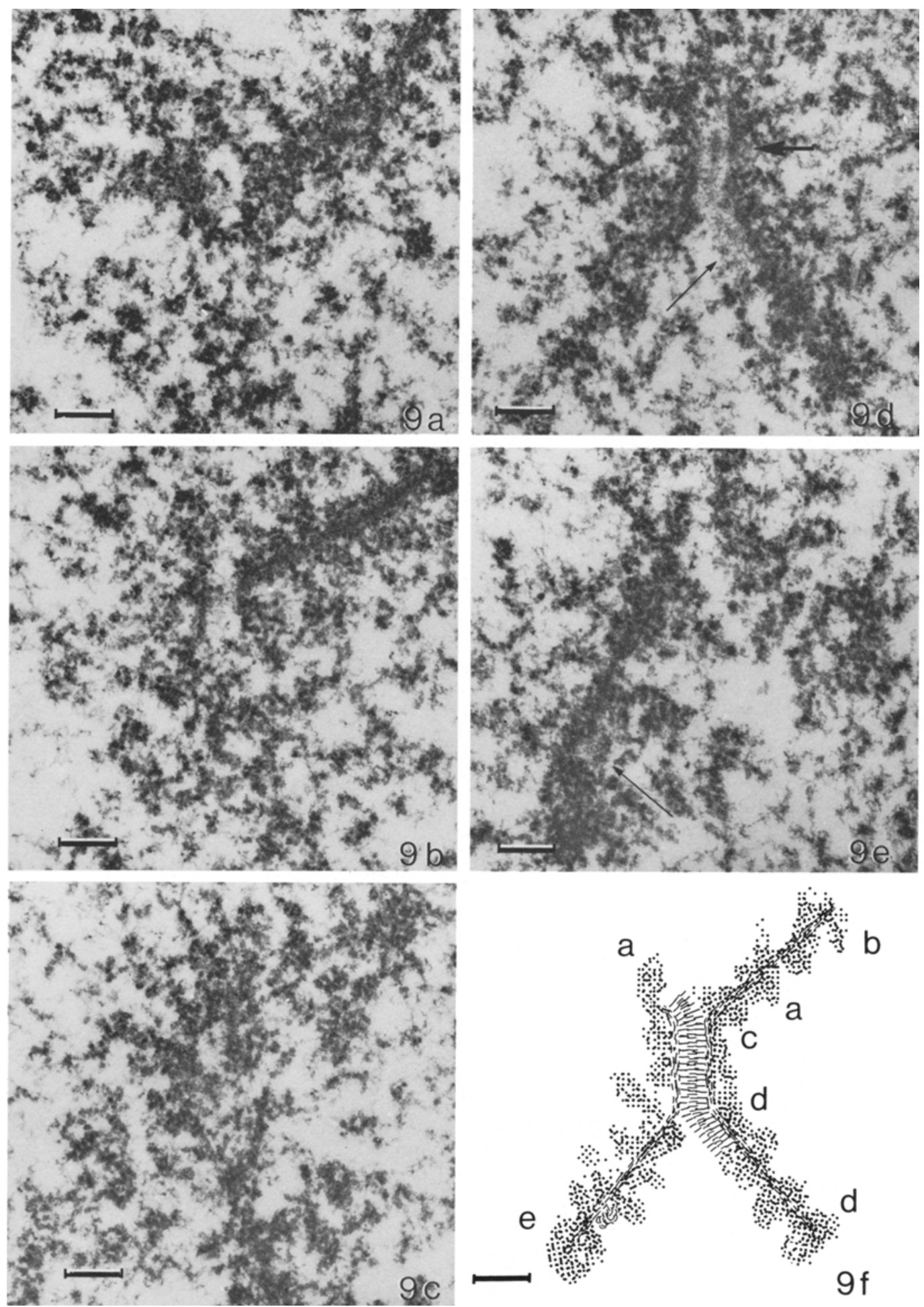

Figure 9: Five consecutive sections of a partially paired bivalent region. In Figure 9d the thin arrow denotes a piece of organized central region associated with only one lateral component. Rotation of the chromatin has occured at this stretch of the chromosome. The thick arrow points to a local thickening of the central component. In Figure 9e the arrow denotes an aggregation of filaments interpreted to be precursor material for the central region. Reconstruction of the bivalent components from Figures $9 \mathrm{a}-9 \mathrm{e}$ is presented in Figure $9 \mathrm{f}$. $9 \mathrm{a}-9 \mathrm{e} \times 40.000(\mathrm{Bar}=0,2 \mu \mathrm{m})$. 
lateral component has been observed 5 times in the studies at high magnification.

\subsection{Reconstruction of chromosomes}

\subsubsection{The karyotype of Lilium longiflorum}

The somatic karyotype of Lilium longiflorum $(\mathrm{n}=12)$ has previously been characterized by Feulgen staining and its $C$ and $Q$ banding properties investigated (22). Due to the very large size of the Lilium genome the present study has been limited to reconstruction of one nucleus. Reconstructions of the 12 partially paired bivalents are presented in Figs. 10-21, and an idiogram denoting chromosome lengths and pairing characteristics is presented in Fig. 22. Length measurements for the paired and unpaired chromose parts are given in Table 1-12. Total length of the chromosomes including relative lengths and centromere index for the zygotene chromosomes as well as the colchicine treated and Feulgen stained somatic chromosomes is compiled in Table 13. A good correlation can be observed between the meiotic and somatic chromosomes for the relative lengths and the centromere indices. With the possible exception of chromosomes 8 and 9 , which are hardly distinguishable, all chromosomes seem to be correctly identified.

\subsubsection{General organization of the early zygotene chromosomes}

The total chromosome length of $7.4 \mathrm{~mm}$ provides a primary indication of the complexity of the internal organization of the nucleus. The average chromosome length is 9-10 times longer than the nuclear diameter, necessitating large scale folding of the chromosomes. Moreover, the overall impression from the reconstructions is that the processes of alignment and pairing are dynamic, with chromosome regions constantly moving around in order to avoid interference from non-homologous chromosomes as well as alignment movements towards the homologous partner. Large asynchrony in the alignment and pairing processes is evident: In chromosome 7 only $2,8 \%$ of the chromosome is paired with a synaptinemal complex, whereas in chromosome 8 as much as $58.2 \%$ of the chromosome length is paired with a complex.

The active process of pairing is in particular evident for the nucleolus organizing chromosomes 3,4 and 7. All 6 nucleolus organizers were associated with the two crescent shaped nucleoli in the 3 mid-leptotene nuclei analysed but

\section{Table XIII}

Chromosome length measurements of Lilium longiflorum.

E.M.: Zygotene measurements from reconstructions, L.M.: light microscopic measurements of somatic metaphase after colchicine treatment.

\begin{tabular}{|c|c|c|c|c|c|c|c|c|c|c|}
\hline \multirow[t]{2}{*}{$\begin{array}{l}\text { Chromosome } \\
\mathrm{nr} \text {. }\end{array}$} & \multicolumn{2}{|c|}{$\begin{array}{l}\text { short arm } \\
\mu \mathrm{m}\end{array}$} & \multicolumn{2}{|c|}{$\begin{array}{l}\text { long arm } \\
\qquad \mu \mathrm{m}\end{array}$} & \multicolumn{2}{|c|}{$\begin{array}{l}\text { total length } \\
\mu \mathrm{m}\end{array}$} & \multicolumn{2}{|c|}{$\begin{array}{c}\text { relative length } \\
\%\end{array}$} & \multicolumn{2}{|c|}{$\begin{array}{r}\text { centromere } \\
\text { index } \%\end{array}$} \\
\hline & E.M. & L.M. & E.M. & L.M. & E.M. & L.M. & E.M. & L.M. & E.M. & L.M. \\
\hline 1 & 187,8 & 8,5 & 258,6 & 12,8 & 446,4 & 21,3 & 12,1 & 13,5 & 42,1 & 39,9 \\
\hline 2 & 125,3 & 6,0 & 257,1 & 11,2 & 382,4 & 17,2 & 10,4 & 10,9 & 42,1 & 34,9 \\
\hline 3 & 58,5 & 2,2 & 264,1 & 11,0 & 322,6 & 13,2 & 8,7 & 8,4 & 18,1 & 16,7 \\
\hline 4 & 38,6 & 1,8 & 205,8 & 8,5 & 244,4 & 10,3 & 6,6 & 6,6 & 15,8 & 17,5 \\
\hline 5 & 33,3 & 1,2 & 245,9 & 10,0 & 279,2 & 11,2 & 7,6 & 7,1 & 11,9 & 10,7 \\
\hline 6 & 27,5 & 1,0 & 274,5 & 10,8 & 302,0 & 11,8 & 8,2 & 7,5 & 9,1 & 8,5 \\
\hline 7 & 36,0 & 2,0 & 331,2 & 12,8 & 367,2 & 14,8 & 9,9 & 9,4 & 9,8 & 15,6 \\
\hline 8 & 25,9 & 1,0 & 283,4 & 12,8 & 309,3 & 13,8 & 8,4 & 8,8 & 8,4 & 7,2 \\
\hline 9 & 21,6 & 0,8 & 291,9 & 12,8 & 313,5 & 13,6 & 8,5 & 8,7 & 7,0 & 5,9 \\
\hline 10 & 10,9 & 0,5 & 216,6 & 8,2 & 227,5 & 8,7 & 6,2 & 5,5 & 4,8 & 5,7 \\
\hline 11 & 7,2 & 0,2 & 230,1 & 9,2 & 237,3 & 9,4 & 6,4 & 6,0 & 3,0 & 2,1 \\
\hline 12 & 4,8 & 0,1 & 257,9 & 11,8 & 262,7 & 11,9 & 7,1 & 7,6 & 1,8 & 1,3 \\
\hline Total length & & & & & 3694,5 & 157,2 & 100,1 & 100,0 & & \\
\hline
\end{tabular}




\section{P. B. HoLm: Chromosome pairing in Lilium}

in this zygotene nucleus the organizers of both chromosomes 3 and the organizer of one chromosome 7 are not attached to the major nucleoli, and are located far away from them. Such distant location of organizers has been observed in a number of other zygotene nuclei which have been investigated. It is possible that this signifies a detachment of organizers from the nucleolus during pairing.

Of special interest is the behaviour of the telomeres. Each chromosome pair has at least two of its telomeres associated with the nuclear envelope. The telomeres of the short arms of chromosomes 3 and 4 are paired with a synaptinemal complex and not attached to the nuclear envelope. Only one of the two unpaired telomeres of the long arms of chromosomes 7 and of the short arms of chromosomes 12 is bound to the nuclear envelope. The two ends of the short arms of chromosomes 9 and those of the long arms of chromosomes 10 are closely associated by their telomeres, in an end to end fashion free in the nucleoplasm. They have not yet formed a synaptinemal complex.

I consider it likely that both the paired and unpaired telomeres, which were unattached to the nuclear envelope in this nucleus at the moment of fixation had previously been in contact with the envelope and that the detachment is a temporary one in order to facilitate pairing and to resolve interlocking.

A comparison of the reconstructions of the 12 chromosomes reveals all stages of alignment between and within chromosomes. In chromosomes $1,3,7$ and 10 and in certain portions of chromosomes 4, 5 and 9 pairing and rough alignment is very limited, the homologous regions often being separated by distances of up to $30 \mu \mathrm{m}$. This is easily seen for the centromeres of chromosomes 1, 5, 7, 9 and 12 as well as for the unpaired telomeres. The reconstructions prove that individual regions of a pair of homologous chromosomes act independently. Synaptinemal complex formation can be completed in one region before rough alignment is commenced in an adjacent one. Supraorganizational patterns for the chromosomes are not generally evident but an unpaired chromosome region may form hairpin loops over a considerable distance, whereby the risk of interlocking with other chromosome regions is reduced (cf. chromosomes 4 and 10). Such an organization is observed for many homologous chromosome regions in the process of alignment (see chromosomes 5, 6 and 11). To what extent such a hairpin organization is a reflection of a prezygotene chromatin organization awaits further clarification.

Chromosome and bivalent interlocking has been described in several Lilium species for diplotene and diakinesis in light microscopical investigations (36), and has recently been demonstrated from three-dimensional reconstructions of the zygotene stage in female Bombyx mori (46). Indications of interlocking have been found in several regions of the present nucleus, but the precise tracing of the lateral components in regions where they pass each other very closely remains equivocal. In the case arrowed in Fig. 14 an interlocking of an unpaired region with a paired part of the same chromosome 5 is possible. Because of the uncertainty it has not been drawn as an interlocking.

Besides the centromeres and the knobs of the NOR, few markers have been observed. Chromosomes 2 and 3 were homozygous for knobs in the long arm. In chromosome 3 this knob is located proximal to the NOR. Heterozygosity for knobs has been observed in the long arms of bivalents 3 and 7 . In bivalent 3 two knobs have been found just distal to the NOR and in bivalent 7 a knob is observed $160 \mu \mathrm{m}$ from the centromere. This knob pattern correlates not very well with the $\mathrm{C}$ banding pattern observed (22).

\subsubsection{Chromosome pairing}

The precision of chromosome pairing has been elucidated by length measurements of the homologous unpaired lateral components of the synaptinemal complex (Tables 1-12). The results demonstrate a close correlation between the length of the unpaired regions also in the very long unpaired stretches in the middle of most chromosomes. Since the length has been measured over ten sections at a time, the measuring error is increasing the more the chromosome deviates from a linear path. This probably accounts for differences observed between the homologous lateral components. If the unpair- 


\section{P. B. HOLM: Chromosome pairing in Lilium}

ed regions follow a nearly linear path identity in lenth was obtained. The possibility that the lateral component and the associated chromatin possess a minor degree of flexibility allowing limited extension and stretching to take uplace cannot be totally excluded, but for the majority of the chromosome regions it is evident that the lateral component fixes the chromosome length

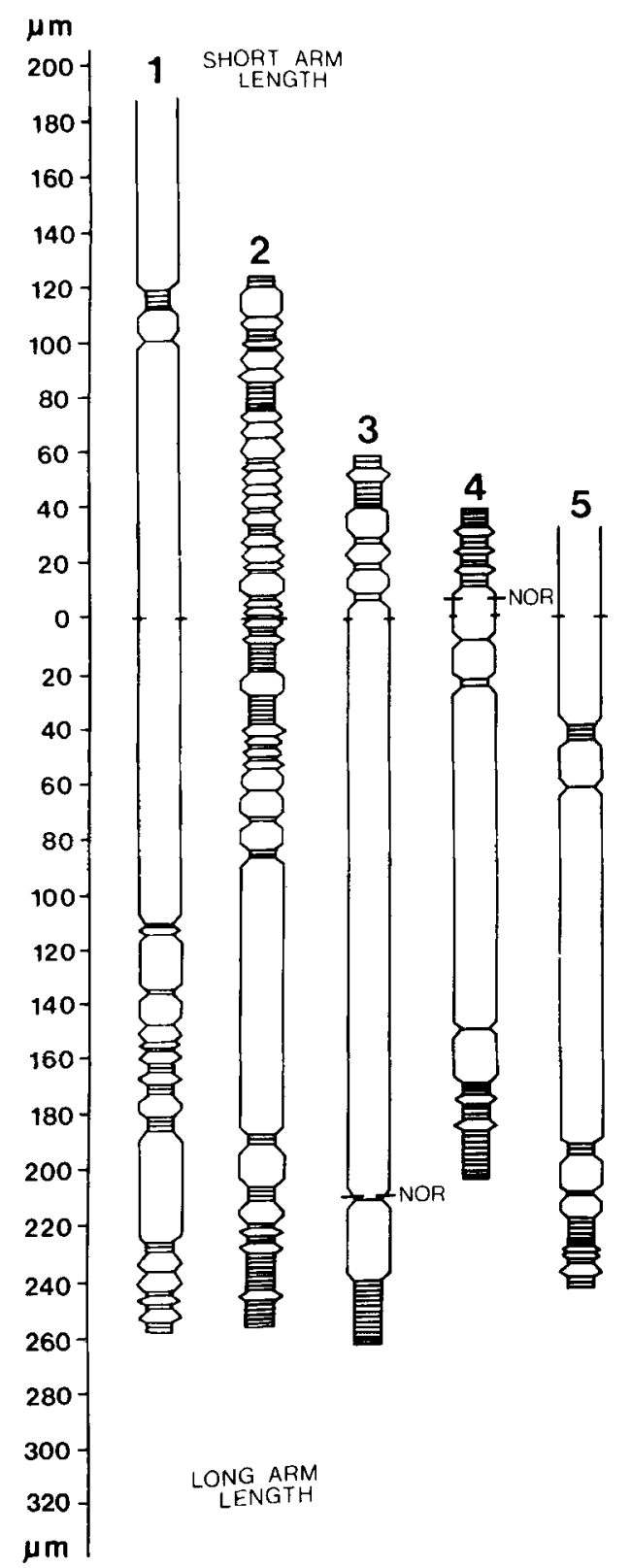

before the precise site-to-site synapsis is initiated.

From the length measurements it can be concluded, that the vast majority of pairing observed is homologous pairing. Three clearcut examples of non-homologous pairing can be demonstrated. Chrosome 4 is paired with chromosomes 7 and 9 over a minor region, and chromosome 6 has formed a synaptinemal complex by a foldback type of pairing with itself.

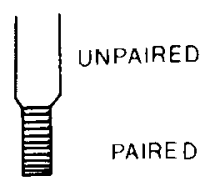

Figure 22: Idiogram of the 12 partially synapsed bivalents of Lilium longiflorum. The length of the arms, centromere position and NOR position as well as the unpaired and paired regions are shown. 


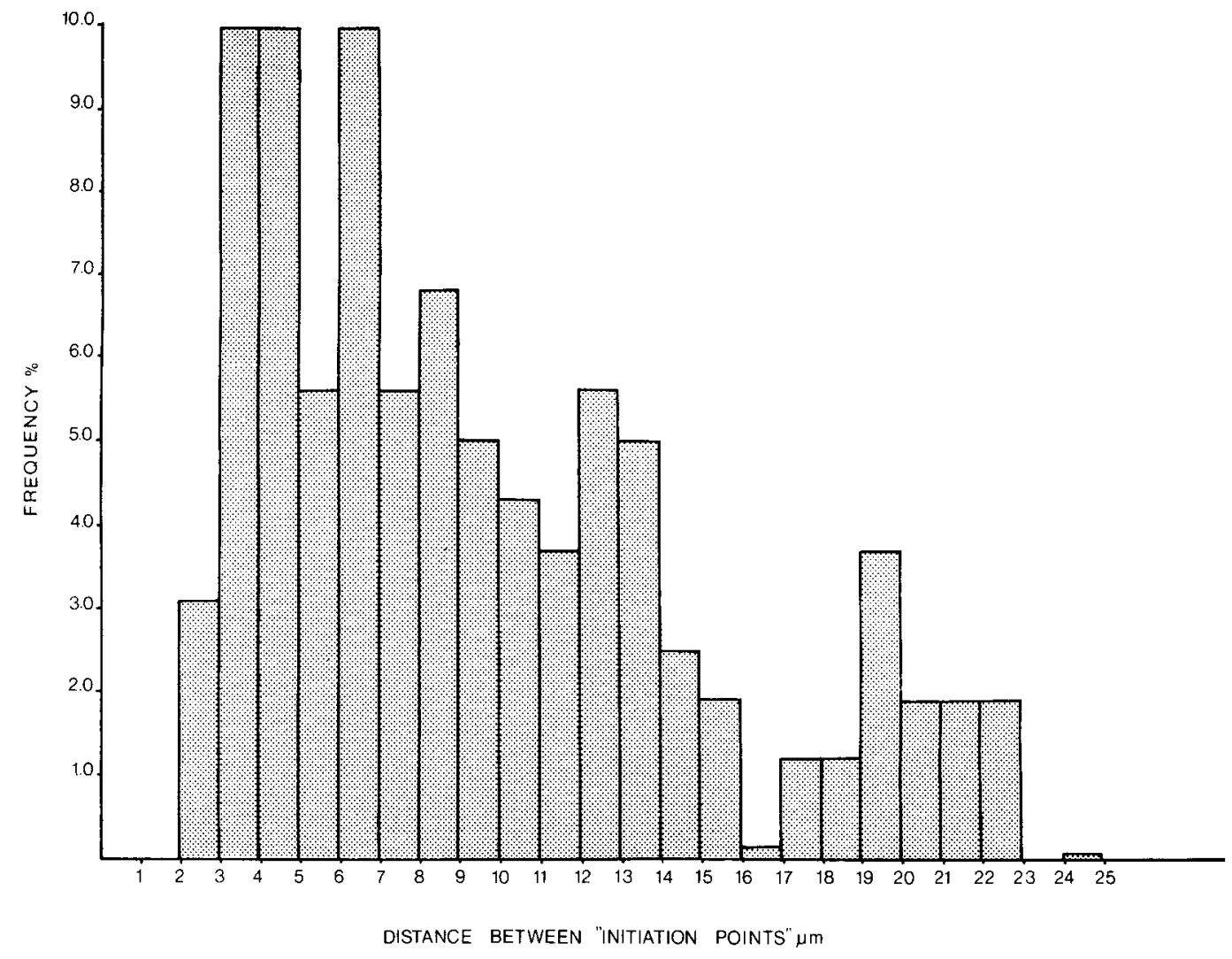

Figure 23: Histogram denoting distances between "initiation points" of pairing, as deduced from distances between completed stretches of synaptinemal complex.

In the calculations it has been assumed that pairing has been initiated in the middle of a stretch of synaptinemal complex. If the telomeres are paired, pairing was assumed to have started from the telomeric end. Distances above $25 \mu \mathrm{m}$ are not shown. They comprise $8 \%$ of the total "initiation point"-to-"initiation point" distances. The frequency is calculated on the basis of the total number of observations.

\subsubsection{Initiation of pairing}

From the present study it is deduced that pairing in lily is initiated at multiple sites along the chromosomes. This refers to pairing by alignment as well as synaptinemal complex formation. As summarized in Fig. 22 the number of synaptinemal complex regons varies from 5 in chromosome 7 to 36 in chromosome 2 . The question whether pairing is initiated at specific and preferred points along the chromosome or whether any part of the chromosome houses the required information for a specific pairing can be approached on the reconstructed nucleus. From Fig. 22 it is evident that telomere regions show more completed synaptinemal complexes than interstitial regions. On the other hand in chromosome 7 complexes are only found at a proximal region of the long arm. Thus, distal regions of the chromosome arms are preferred regions of initiation but any region seems capable of initiating complex formation. An analysis of the distances between the "initiation points" for pairing along the chromosomes is presented in Fig. 23, assuming that synaptinemal complex formation is initiated at the nuclear envelope and in the middle of the complexes in the other portion of the chromosomes. The distances vary between $2,5 \mu \mathrm{m}$ and over $25 \mu \mathrm{m}$. Initiation does not seem to commence at regular intervals along the chromosomes. Centromeres do not pair precociously, but rather belong to the regions in which 
synaptinemal complex formation occurs late. Non-homologous centromere fusion has only been observed once, the unpaired centromere of chromosome 7 being associated with the paired centromeres of chromosomes 8 .

\section{DISCUSSION}

\subsection{Nuclear polarity}

The polarized distribution of telomeres in the bouquet arrangement of zygotene nuclei is known to be a characteristic of animal and some plant meioses (10). Reconstructions from electron micrographs of serial sections have revealed that during the bouquet stage at leptotene and zygotene the telomeres are bound to a restricted region of the inner membrane of the nuclear envelope and that the attached telomeres are being redistributed evenly across the envelope at the beginning of pachytene $(38,46)$. GILLIES (19) has demonstrated for maize that also in a higher plant the telomeres of the chromosomes at leptotene and zygotene are attached to a restricted area of the nuclear envelope. Lilium longiflorum has been regarded as an example for the absence of a bouquet organization in early zygotene $(10,36)$. Moreover the binding of telomeres to the nuclear envelope has been questioned $(37,39)$. In the nucleus reconstructed in this study 34 of the 48 telomeres are attached to the inner membrane of the envelope within one-eighth of the nuclear surface. Thus like in maize, lily chromosomes form a bouquet configuration in the early synaptic stages, adding to the evidence that this arrangement is universal and of significance for the primary recognition and pairing of homologous chromosomes. The long and folded nature of many higher plant chromosomes obscures the recognition of their polarized attachment in light microscope preparations.

An additional characteristic of the early zygotene stage in Lilium longiflorum is the polarized distribution of nucleoli and nuclear pores which is first evident in late leptotene. Pores are absent over the appressed nucleoli, a phenomenon that has been demonstrated in several plants (35). In mid leptotene only a few telomeres are associated with the nuclear membrane, while the late leptotene nucleus posses- ses a well defined bouquet region within and between the major pore regions. These observations reveal that the polarization of the telomeres of early zygotene is not a reflection of their organization during the previous telophase or interphase, but is created by an active process of rearrangement of telomeres, nucleoli and pores.

\subsection{Chromosome length}

A major question in the interpretation of how homologous chromosomes recognize each other and pair in a way that allows precise siteto-site matching is the control and organization of the leptotene chromosome. Cytochemical and enzyme digestion studies have favoured the view that the lateral components formed during leptotene house the required information for precise pairing in the form of RNA and protein associated with a minor DNA fraction $(13,17$, 61). Length measurements of pachytene chromosomes have demonstrated that only a minor fraction of the total DNA can be associated with the lateral component. In Drosophila (34, 62), Neurospora $(16,62)$ Zea mays (17) and Bombyx females (46) the total length of the lateral components are only $0,2 \%, 0,3 \%$, $0,014-0,017 \%$ and $0,12 \%$ respectively of the total DNA length. HOTTA and STERN (29) estimated the $4 \mathrm{C}$ amount of DNA in Lilium longiflorum to be $2,4 \times 10^{14}$ dalton corresponding to $120 \mathrm{~m}$ of extended DNA double helix. The length of the lateral components $(7,4 \mathrm{~mm})$ is accordingly $0,006 \%$ of the DNA length.

Equal length of the lateral component of the synaptinemal complex in unpaired chromosome regions, as found in the present reconstructions, supports the notion that the continuous lateral components determine chromosome length at meiotic prophase until the end of pachytene. This is in agreement with the proposal by GILLIES (19) and RASMUSSEN (46) that major changes in total chromosome length during zygotene and pachytene are not expected. Large changes in chromosome length have been reported during meiotic prophase in Drosophila (5). The constant somatic pairing in Drosophila may account for this special finding. Several investigations have described the lepto- 
tene chromosome as lampbrush-like $(7,9,60)$, but the molecular nature of the attachment of the chromatin from the two sister chromatids to the lateral component is not understood. A meiosis-unique histone with some similarities to the $\mathrm{H} 1$ histone is synthesized during the preleptotene-leptotene interval in Lilium longiflorum (50) and total histone synthesis has been reported to be uncoupled from the premeiotic DNA replication and to extend into zygotene $(3,56)$. These observations suggest that the organization of the leptotene chromosome and the components of the synaptinemal complex might be related to the formation of the specific meiotic histone. It has been suggested $(23,54)$ that the extended premeiotic S-phase is of importance for the control of the organization of the genome and for a preselection of regions to engage in crossing over during pachytene (55). In Lilium longiflorum the premeiotic S-phase has been estimated to be six times longer than the average somatic S-phase and an interval of 8-10 hours has been found between the DNA replication in euchromatin and heterochromatin. The organization of the leptotene chromosome is thus integrated with a distinct temporal sequence of the premeiotic DNA replication in different chromosome regions. Other lines of evidence suggest that the premeiotic DNA synthesis is a controlling factor for the organization of the leptotene chromosome. In a series of investigations HOTTA, STERN and coworkers have demonstrated, that DNA replication of $0,3 \%$ of the genome is delayed until zygotene $(25,28)$. This DNA consists of unique sequences (29), is scattered throughout the genome (31), characterized by a $\mathrm{G}+\mathrm{C}$ content of $50 \%(25,28)$ and has an average length of 3500 basepairs (29). Inhibition of this zygotene-DNA synthesis interrupts the formation of the synaptinemal complex (48). It has been observed that no single stranded regions occur during leptotene, indicating that the two chromatids share the same double helix in the unreplicated regions (30), and that the DNA regions replicated during zygotene are not ligated to the flanking DNA before pachytene (30). These observations have led to the conclusion, that the zygotene-DNA regions serve as primary recognition sites for the homologous chromosomes. No evi- dence is yet available on the localization of the zygotene-DNA with respect to the lateral components of the synaptinemal complex and the relationship of its replication to the morphology of the formation of the synaptinemal complex. If arranged along the lateral components it must be packed by a factor 50 since the total length of the lateral components is only $0,006 \%$ of the DNA length and the zygotene DNA comprises $0,3 \%$.

\subsection{Chromosome alignment and pairing}

The mechanism by which chromosomes align and pair is still under debate. STERN \& HOTTA (54) favour the hypothesis that pairing is specified at the DNA level through matching of homologous regions of zygotene-DNA, followed by alignment and ultimate organization of the synaptinemal complex via the formation of the central region. WESTERGAARD \& VON WETTSTEIN $(61,62)$ and VON WETTSTEIN $(63,64)$ suggested that homologous chromosomes are roughly aligned by some unknown mechanism and that the required specificity for pairing was housed in the form of RNA and protein in the lateral components. It was hypothesized (63) that by binding of the central region to one of the lateral components a series of conformational changes was induced in the unspecific central region, which created a specificity on the opposite surface of the central region matching the lateral component of the homologous chromosome. The location of homologous chromosomes in the prezygotene nuclei has been discussed for several years. From light microscopical observations some authors have deduced various degrees of somatic pairing of homologous chromosomes (4), or associations between homologous telomeres (58). COMINGS \& OKADA (7) observed closely associated telomeres in waterspread leptotene nuclei, and argued that this organization also was a characteristic of somatic nuclei. The light microscope observations have been disputed and criticised repeatedly (cf. 32). With the exception of Diptera the three-dimensional reconstructions of leptotene and zygotene nuclei prove the absence of pre-alignment. Also telomeres are found to be separated at leptotene by distances several to many times greater than their ulti- 


\section{P. B. HOLM: Chromosome pairing in Lilium}

mate distance within the synaptinemal complex $(19,38,46)$. These results are in agreement with light microscope observations from Lilium longiflorum (59). The present reconstruction study provides definitive evidence against premeiotic alignment of homologous chromosomes in this organism.

Telomere migration seems mediated by their movements in or close to the nuclear envelope, since telomeres are attached to its inner membrane from leptotene to pachytene. Membranes of the nuclear envelope have been repeatedly considered as transport vehicles for chromosomes $(39,42)$. RASMUSSEN $(46)$ favoured the idea that the resolution of the bouquet configuration at late zygotene in Bombyx is mediated by sliding movements of the inner membrane as signified by the formation of nuclear membrane vacuoles. In Lilium longiflorum synthesis of lipoprotein material has been observed during the leptotene-zygotene period (STERN pers. commun.). The invaginations of the nuclear envelope observed in the present study and telomere movements may be related to this synthesis. The filaments traversing the perilacunar space $(14,60$ and this investigation) at the site of telomere attachment or modified nuclear pores (11, 15) can be envisaged to play a role in telomere movement. The alignment and movement of the telomeres cannot by itself bring about the rough alignment of $7,4 \mathrm{~mm}$ of chromosome length in a nucleus with a diameter of $0,03 \mathrm{~mm}$. Whether or not the association of interstitial chromatin to the nuclear envelope through dense plates, the special substance (this study), fibers discovered in wheat (2), nuclear pores $(8$, 9) or an organizational matrix in the nucleoplasm (33) promote alignment of the homologues, remains to be analyzed.

From a structural point of view the formation of the synaptinemal complex in lily is characterized by two major events, i. e., rotation of the chromatin with respect to the lateral component of one of the homologous chromosomes followed by the binding of the lateral component to the preformed central region. This pairing mechanism is identical to the one proposed by WESTERGAARD \& VON WETTSTEIN $(61,63)$ and proven by VON WETTSTEIN (64) through reconstruction of a zygotene nucleus in Neottiel- la. It implies that the central region cannot be an integral part of the zygotene chromosome or the lateral component as often proposed $(8,9$, 37). Investigations in Neottiella $(61,64)$ and Drosophila $(43,44,45)$ have suggested that either synthesis or temporary assembly of the central region material takes place in the nucleolus prior to pairing with subsequent transport to the chromosomes in an amorphous form. Organized central region material has not been observed in the nucleolus of lily.

The DNA replicated during zygotene is transiently associated with a lipoprotein complex (21), and a protein similar to the gene-32 protein of the phage T4, which facilitates the renaturation of single stranded DNA, has been isolated from a heavy membrane fraction (26, 27). In addition a colchicine binding protein has been isolated from the same fraction, this protein is identical in its response to colchicine as tubulin but with a lower molecular weight and insensitive to vinblastine (24). Since colchicine treatment of zygotene meiocytes in Lilium interferes with pairing and reduces chiasma frequency (49), STERN \& HOTTA (54) concluded that the nuclear lipoprotein and the gene-32 protein provide the framework for molecular recognition of homologous chromosomes via the zygotene-DNA regions and moreover, that the primary meiotic effect of colchicine is an inhibition of one or more of the steps in the pairing process. The establishment of causal relations between the biochemical and the ultrastructural characteristics of chromosome pairing require further links than are available at present. One observation may provide such a link: Before the zygotene-DNA is replicated it can be assumed that the two sister chromatids are not individualized and the shared DNA stretches are packed around the lateral component. The zygotene-DNA replication is conceivably the event that triggers the rotation of the two chromatids with respect to the lateral component, thereby exposing it for binding with the central region.

\subsection{Initiation of pairing}

Three major hypotheses have been put forward on the nature of the initiation of pairing. KING (34) suggested that pairing was accom- 


\section{P. B. HoLM: Chromosome pairing in Lilium}

plished by specific initiation points at the telomeres followed by an unspecific zipping up for the rest of the chromosome. COMINGS \& OKADA $(8,9)$ favoured the idea of multiple initiation points with unspecific zipper mechanisms accounting for the pairing of the rest of the genome. WESTERGAARD and VON WETTSTEIN (62) argued that information for specific site-to-site pairing was housed all along the lateral component.

Reconstruction studies have revealed that pairing in some organisms proceeds regularly from the telomeres, without any initiation in the internal parts of the chromosomes (46). In Zea mays it was noted, however, that pairing also was initiated in the internal parts of the chromosomes (19), and a previous (37) and this investigation of Lilium longiflorum have demonstrated the same phenomenon. The pairing behaviour observed in inversion heterozygotes (17), translocation heterozygotes (52) and sex chromosomes in the heterogametic sex (51) is difficult to explain unless specificity for pairing is housed all along the chromosomes.

\subsection{Non-homologous pairing}

Three possible cases of non-homologous pairing have been observed. Due to limited information on the evolution of the Lilium longiflorum genome it cannot be stated definitively if really non-homologous regions are paired. Nonhomologous pairing has been observed in several studies, and reconstructions of such pairing at the ultrastructural level are available for haploids $(18,57)$ as well as for the $K_{10}$ chromosome of Zea mays (17). The mechanism behind such pairing events remains obscure, but it might be visualized as follows: The binding of pieces of the central region occurs initially to one or the other of the lateral components. Absence of homologous lateral components might still lead to complex formation if the blocks of organized central regions have a strong tendency to aggregate in the longitudinal direction. Recent reconstructions from triploid Bombyx females (47) reveal that even after formation of synaptinemal complexes paired chromosome regions can separate again and pair in a different way with synaptinemal complex for- mation. The nonhomologous complexes observed in this zygotene nucleus are considered to be temporary and prone to correction.

\section{ACKNOWLEDGEMENTS}

I am indebted to the late Professor Mogens WESTERGAARD for suggesting this project and for his helpful guidance. I would also like to thank Professor Herbert Stern, Professor DITER VON WETTSTEIN and lic. scient SøREN W. RASMUSSEN for valuable discussions and comments on the manuscript. The technical assistance of Ms. JEAN SAGE is gratefully acknowledged. The staff of the phytotron at the Royal College of Forestry in Stockholm are thanked for their care of the plants. This work was supported in part by grant GM-22051 of the National Institutes of Health, U. S. Public Health Service to Professor D. vON WETTSTEIN.

\section{REFERENCES}

1. Anderson, P. J.: Purification and quantitation of glutaraldehyde and its effect on several enzyme activities in skeletal muscle. J. Histochem. Cytochem. 15, 652-661 (1967)

2. BenNetT, M. D., H. STeRn \& M. Woodward: Chromatin attachment to the nuclear membrane of wheat pollen mother cells. Nature 252, 395-396 (1974)

3. Bogdanow, Yu, F., A. A. Strokov \& S. A. RESNIKOVA: Histone synthesis during meiotic prophase in Lilium. Chromosoma (Berl.) 43, 237245 (1973)

4. BROWN, W. V. \& S. M. STACK: Somatic pairing as a regular preliminary to meiosis. Bull. Torrey Bot. Club 95, 369-378 (1964)

5. CARPENTER, A. T. C.: Electron microscopy of meiosis in Drosophila melanogaster females. I. Structure, arrangement and temporal change of the synaptonemal complex in wild type. Chromosoma (Berl.) 51, 157-182 (1975)

6. Carpenter, A. T. C.: Electron microscopy of meiosis in Drosophila melanogaster females: II: The recombination nodule- a recombination associated structure at pachytene? Proc. Nat. Acad. Sci. 72, 3186-3189 (1975)

7. Comings, D. E. \& T. A. OKadA: Whole mount 


\section{P. B. HoLm: Chromosome pairing in Lilium}

electron microscopy of meiotic chromosomes and the synaptonemal complex. Chromosoma (Berl.) 30, 269-286 (1970)

8. Comings, D. E. \& T. A. OKADA: Mechanisms of chromosome pairing during meiosis. Nature 227, 451-456 (1970)

9. Comings, D. E. \& T. A. OKaDA: Architecture of meiotic cells and mechanisms of chromosome pairing. Adv. Cell. Mol. Biol. 2, 310-384 (1972)

19. Darlington, C. D.: Recent Advances in Cytologi. 2nd ed. Churchill, London 671 pp. (1937)

11. Engelhardt, P. \& K. Pusa: Nuclear pore complexes: 'Press-stud"' elements of chromosomes in pairing and control. Nature New Biol. 240, 163-166 (1972)

12. ERICKSON, R. O.: Cytological and growth correlations in the flower bud and anther of Lilium longiflorum. Am. J. Bot. 729-739 (1948)

13. ESPONDA, P. \& J. C. STOCKERT: Localization of RNA in the synaptinemal complex. J. Ultrastruct. Res. 35, 411-417 (1971)

14. Esponda, P. \& G. Gimenez-Martin: The attachment of the synaptinemal complex to the nuclear envelope. Chromosoma (Berl.) 38, 405-417 (1972)

15. Fill, A. \& P. B. MoENs; The development, structure and function of modified synaptonemal complexes in mosquito oocytes. Chromosoma (Berl.) 41, 37-62 (1973)

16. Gillies, C. B.: Reconstruction of the Neurospora crassa pachytene karyotype from serial sections of synaptonemal complexes. Chromosoma (Berl.) $36,119-130(1972)$

17. GILLIES, C. B.: Ultrastructural analysis of maize karyotypes by three-dimensional reconstructions of the synaptonemal complexes. Chromosoma (Berl.) 43, 145-176 (1973)

18. Gillies, C. B.: The structure and the extent of synaptonemal complex formation in haploid barley. Chromosoma (Berl.) 48, 441-453 (1974)

19. Gillies, C. B.: An ultrastructural analysis of chromosome pairing in maize. Compt. Rend. Trav. Lab. Carlsberg 40, 135-161 (1975)

20. Gillies, C. B.: Synaptonemal complex and chromosome structure. Ann. Rev. Genet. 9, 91-109 (1975)

21. HECHT, N. B. \& H. STERN: A late replicating DNA-protein complex from cells in meiotic prophase. Exp. Cell. Res. 69, 1-10 (1971)

22. Holm, P. B.: The $\mathrm{C}$ and $\mathrm{Q}$ banding patterns of the chromosomes of Lilium longiflorum (Thunb.). Carlsberg Res. Commun. 41, 217-224 (1976)

23. Holm, P. B.: The premeiotic DNA replication of euchromatin and heterochromatin in Lilium longiflorum (Thunb.). Carlsberg Res. Commun. (in preparation)
24. HotTA, Y. \& Y. SHEPHARD: Biochemical aspects of colchicine action on meiotic cells. Molec. Gen. Genet. 122, 243-260 (1973)

25. Hotta, Y., M. ITo \& H. STERN: Synthesis of DNA during meiosis. Proc. Nat. Acad. Sci. 56, 1184-1191 (1966)

26. HotTA, Y. \& H. STERN: A DNA binding protein in meiotic cells of Lilium. Dev. Biol. 26, 87-99 (1971)

27. HotTA, Y. \& H. STERn: Meiotic protein in spermatocytes of mammals. Nature New Biol. 234, 83-86 (1971)

28. HotTA, Y. \& H. STERN: Analysis of DNA synthesis during meiotic prophase in Lilium. J. Mol. Biol. 55, 337-355 (1971)

29. HotTA, Y. \& H. STERN: Zygotene and pachytenelabelled sequences in the meiotic organization of chromosomes. In: The eukaryotic chromosome, W. S. Peacock \& R. D. Brock eds., Austral. Nat. Univ. Press Canberra pp 283-300 (1975)

30. Hotta, Y. \& H. Stern: Persistent discontinuities in late replicating DNA during meiosis in Lilium. Chromosoma (Berl.) 55, 171-182 (1976)

31. ITo, M. \& H. STERN: Radioautography of incorporated ${ }^{3} \mathrm{H}$-thymidine and its metabolism during meiotic prophase in microsporocytes of Lilium. Chromosoma (Berl.) 43, 391-398 (1973)

32. JoHN, B.: Myths and mechanism of meiosis. Chromosoma (Berl.) 54, 295-325 (1976)

33. Keller, J. M. \& D. E. Riley: Nuclear ghosts: A non-membranous structural component of mammalian cell nuclei. Science 193, 399-401 (1976)

34. KING, R. C.: The meiotic behaviour of the Drosophila oocyte. Int. Rev. Cytol. 28, 125-168 (1970)

35. LACOUR, L. F. \& B. WELLS: The nuclear pores of early meiotic prophase nuclei of plants. Z. Zellforsch. 123, 178-194 (1972)

36. Mather, K: Meiosis in Lilium. Cytologia 6, 354-380 (1935)

37. MOENS, P. B.: The structure and function of the synaptinemal complex in Lilium longiflorum sporocytes. Chromosoma (Berl.) 23, 418-451 (1968)

38. MOENS, P. B.: The fine structure of meiotic chromosome polarization and pairing in Locusta migratoria spermatocytes. Chromosoma (Berl.) 28, 1-25 (1969)

39. Moens, P. B.: Mechanisms of chromosome synapsis at meiotic prophase. Int. Rev. Cyt. 35, 117-134 (1973)

40. MoENS, P. B.: Quantitative electron microsopy of chromosome organization at meiotic prophase. Cold. Spr. Harb. Symp. Quant. Biol. 38, 99-107 (1973)

41. Moses, M. J.: Synaptinemal complex. Ann. Rev. Genet. 2, 363-412(1968) 


\section{P. B. HoLm: Chromosome pairing in Lilium}

42. PicketT-Heaps, J. D.: The evolution of the mitotic apparatus: An attempt at comparative ultrastructural cytology in dividing plant cells. Cytobios. 3 , 257-280 (1969)

43. Rasmussen, S. W.: Ultrastructural studies on spermatogenesis in Drosophila melanogaster Meigen. Z. Zellforsch. 140, 125-144 (1973)

44. Rasmussen, S. W.: Studies on the development and ultrastructure of the synaptinemal complex in Drosophila melanogaster. Compt. Rend. Trav. Lab. Carlsberg 39, 443-468 (1974)

45. RASMUSSEN, S. W.: Ultrastructural studies of meiosis in males and females of the $c(3) \mathrm{G}^{17}$ mutant of Drosophila melanogaster. Compt. Rend. Trav. Lab. Carlsberg 40, 163-173 (1975)

46. Rasmussen, S. W.: The meiotic prophase in Bombyx mori females analyzed by three-dimensional reconstructions of synaptonemal complexes. Chromosoma (Berl.) 54, 245-293 (1976)

47. Rasmussen, $S$. W.: Chromosome pairing in triploid females of Bombyx mori analyzed by threedimensional reconstruction of synaptonemal complexes. Carlsberg Res. Commun 42, (1977)

48. Roth, I. F. \& M. ITO: DNA dependent formation of the synaptinemal complex. J. Cell. Biol. 35, 247-255 (1967)

49. SHEPHARD, W. F., E. R. BOOTHROYd \& H. STERN: The action of colchicine on synapsis in meiotic cells of Lilium. Chromosoma (Berl.) 44, 423-437 (1974)

50. Sheridan, W. F. \& H. Stern: Histones of meiosis. Exp. Cell Res. 45, 323-335 (1967)

51. SOLARI, A. J. \& L. L. TREs: The three-dimensional reconstruction of the $X Y$ chromosomal pair in human spermatocytes. J. Cell Biol. 45, 43-53 (1970)

52. SolaRI, A. J.: The behaviour of chromosomal axes in Searles's X-autosome translocation. Chromosoma (Berl.) 34, 99-112 (1971)

53. SPURR, A. R.: A low-viscosity epoxy resin embedding medium for electron microscopy. J. Ultra- struc. Res. 26, 31-43 (1969)

54. Stern, H. \& Y. HotTa: Biochemical controls of meiosis. Ann. Rev. Genet. 7, 37-66 (1973)

55. Stern, H., M. WestergaArd \& D. voN WetTstein: Presynaptic events in meiocytes of Lilium longiflorum and their relation to crossingover: A preselection hypothesis. Proc. Nat. Acad. Sci. 72, 961-965 (1975)

56. Strokov, A. A., Yu. F. Bogdanov \& S. A. REZNIKOVA: A quantitative study of histone of meiocytes II, Polyacrylamide gel electrophoresis of isolated histones from Lilium microsporocytes. Chromosoma (Berl.) 43, 247.260 (1973)

57. TING, Y. C.: Synaptonemal complex of haploid maize. Cytologia 38, 497-500 (1973)

58. WagenaAR, E. B.: End-to-end attachments in mitotic interphase and their possible significance to meiotic chromosome pairing. Chromosoma 26, 410-426 (1969)

59. WALTERS, M. S.: Evidence on the time of chromosome pairing from the preleptotene spiral stage in Lilium longiflorum "Croft". Chromosoma (Berl.) 29, 375-418 (1970)

60. Welsch, B.: Synaptonemal Complex und Chromosomenstruktur in der achiasmatischen Spermatogenese von Panorpa communis (Mecoptera). Chromosoma (Berl.) 43, 19-74 (1973)

61. WestergaARD, M. \& D. von WeTtstein: Studies on the mechanism of crossing-over IV. The molecular organization of the synaptinemal complex in Neottiella (Cooke) Saccardo (Ascomycetes). Compt. Rend. Trav. Lab. Carlsberg 37, 239-268 (1970)

62. WestergaARD, M. \& D. von WetTSTEIN: The synaptinemal complex. Ann. Rev. Genet. 6, 71-110 (1972)

63. Wettstein, D. von: The synaptinemal complex and four-strand crossing over. Proc. Nat. Acad. Sci. 68, 851-855 (1971)

64. WetTSTEIN, D. von: The assembly of the synaptinemal complex. Phil. Trans. R. Soc. Lond. B. 277, 235-243 (1977) 

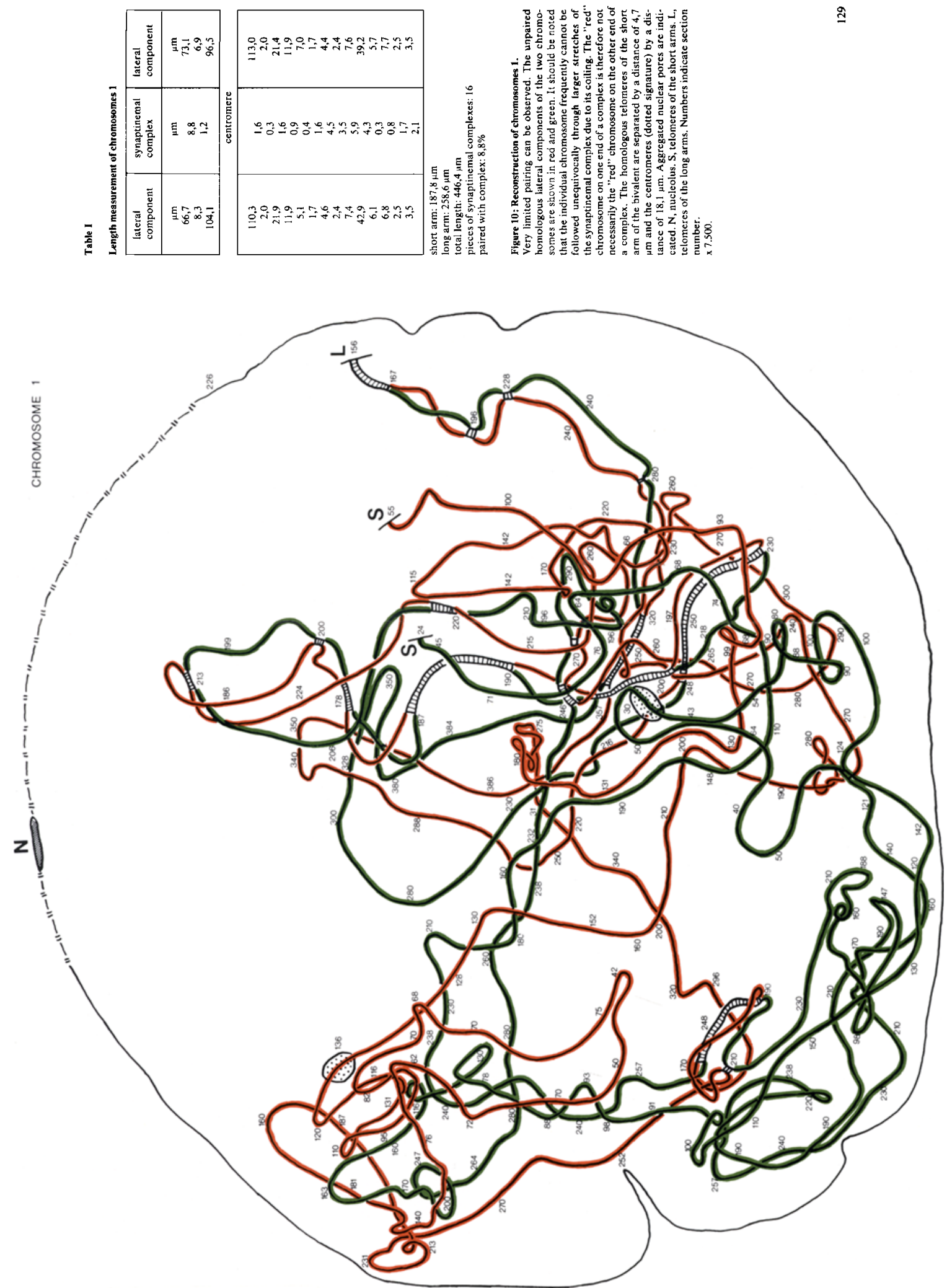

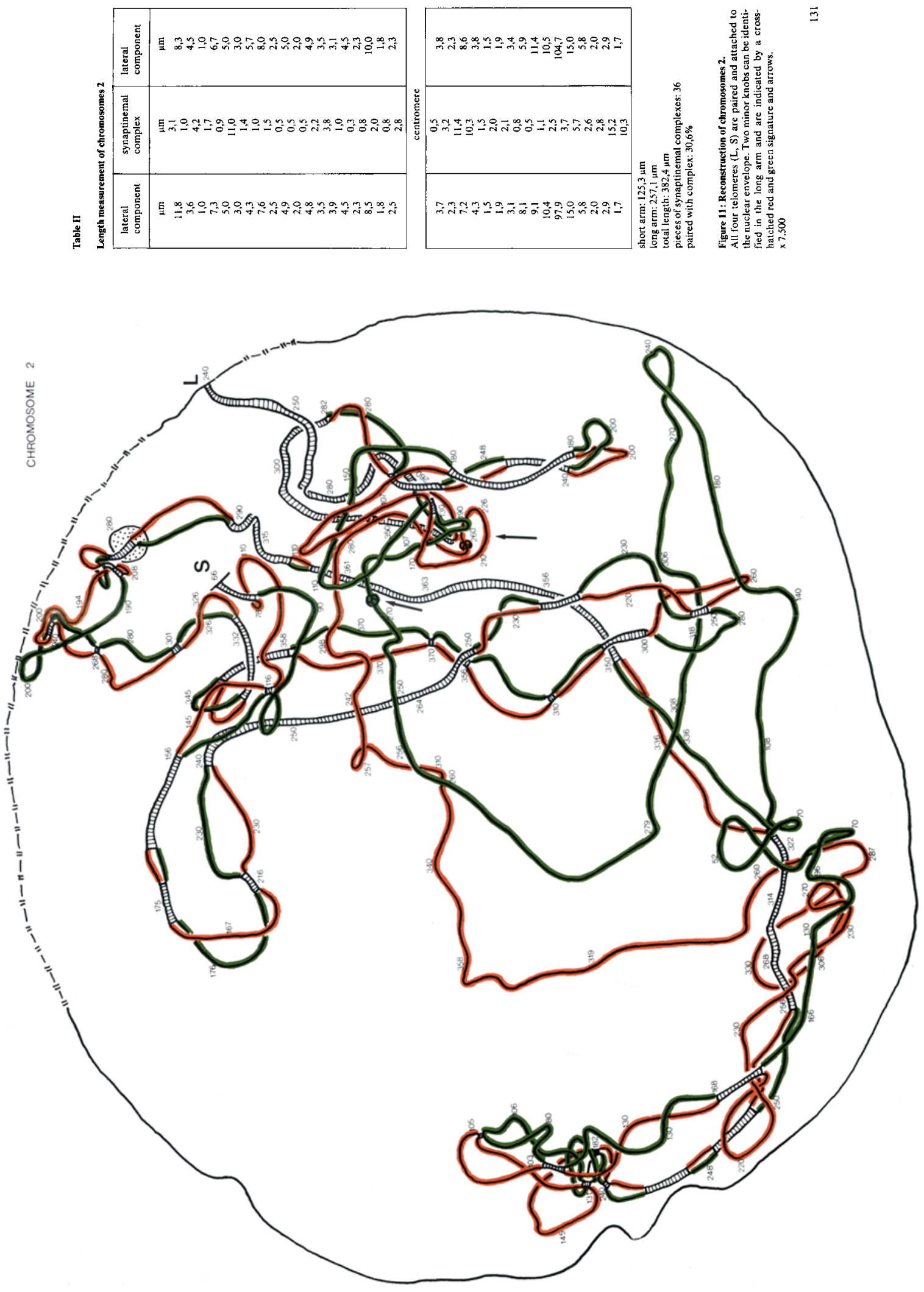


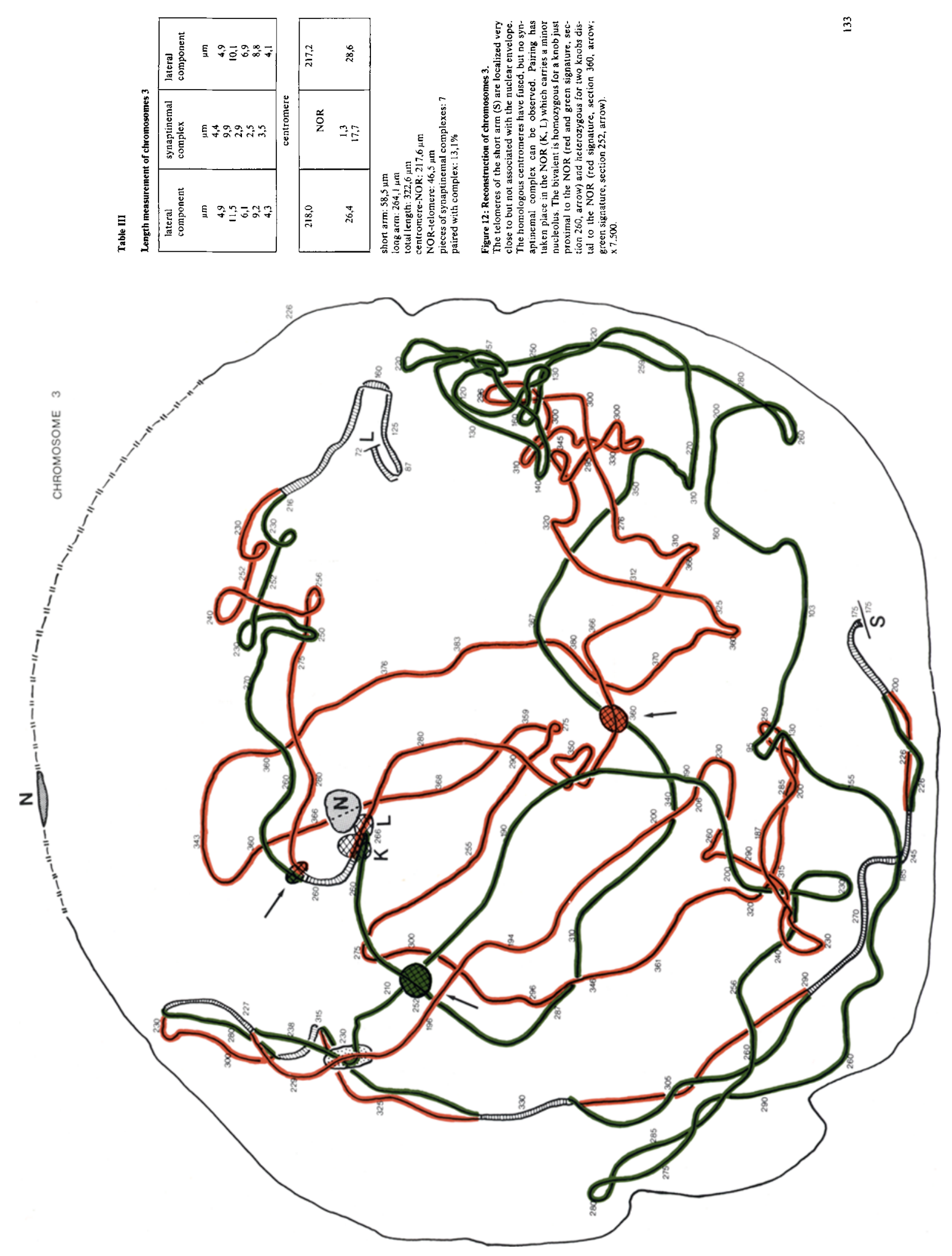



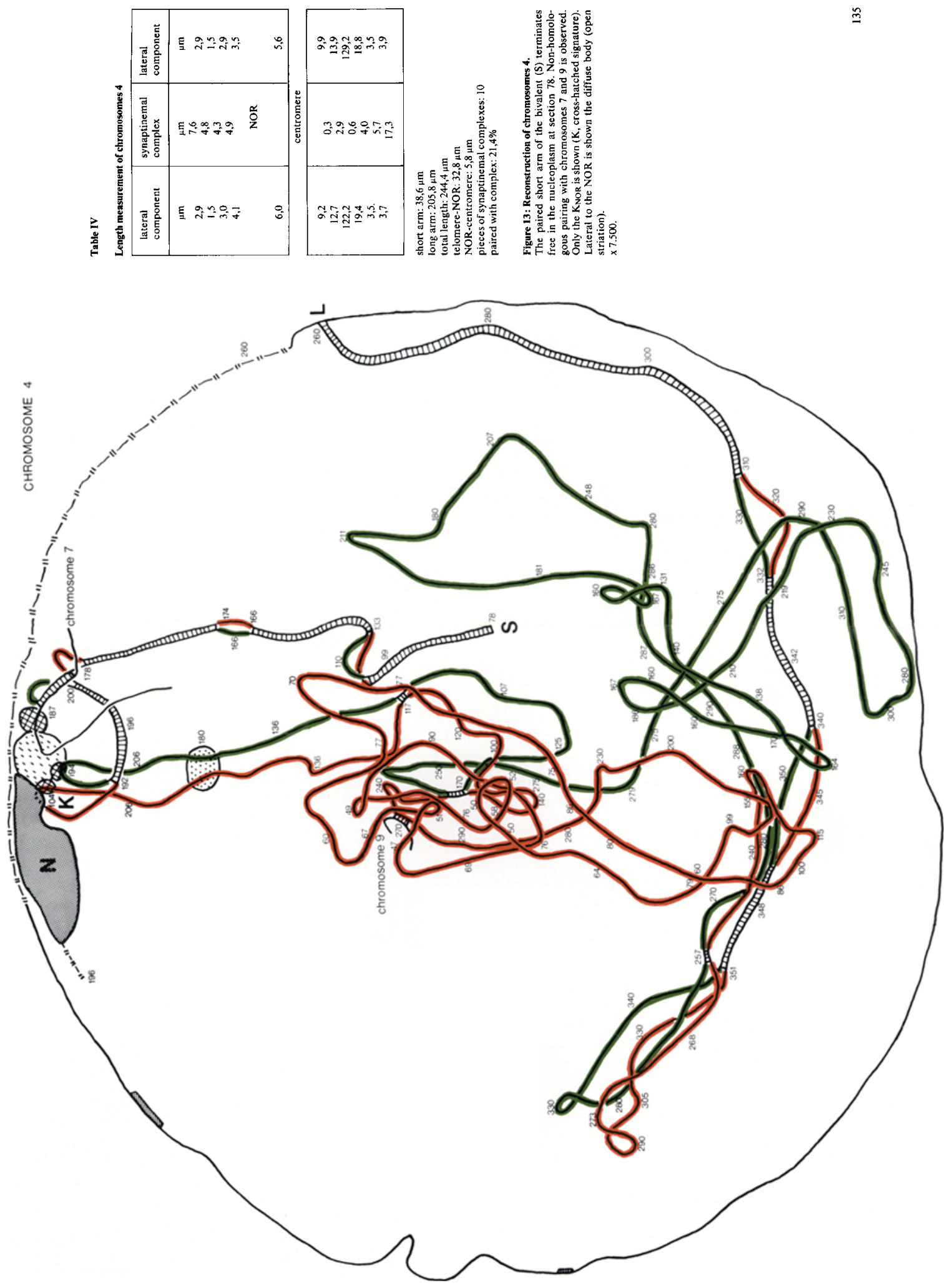

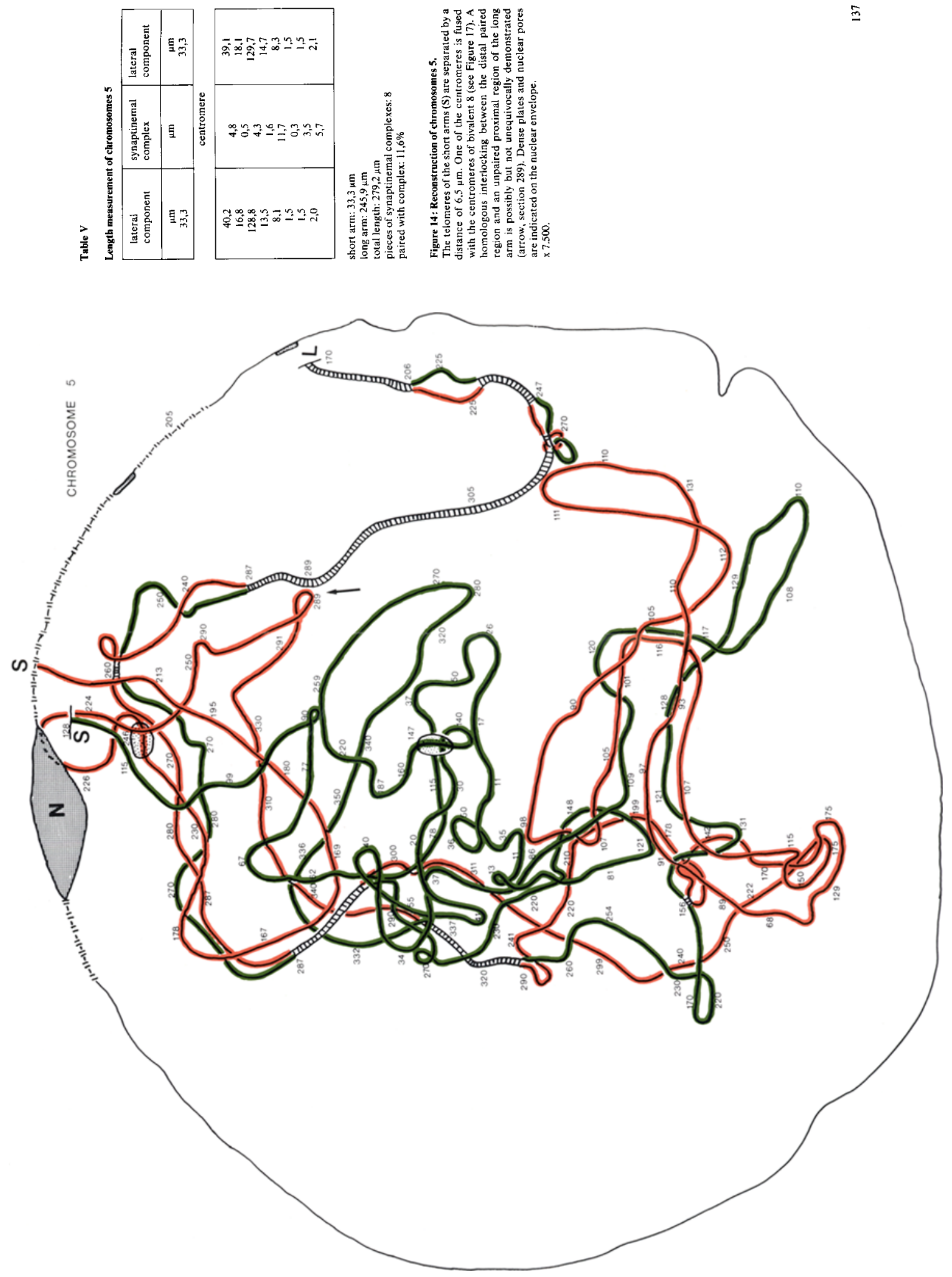

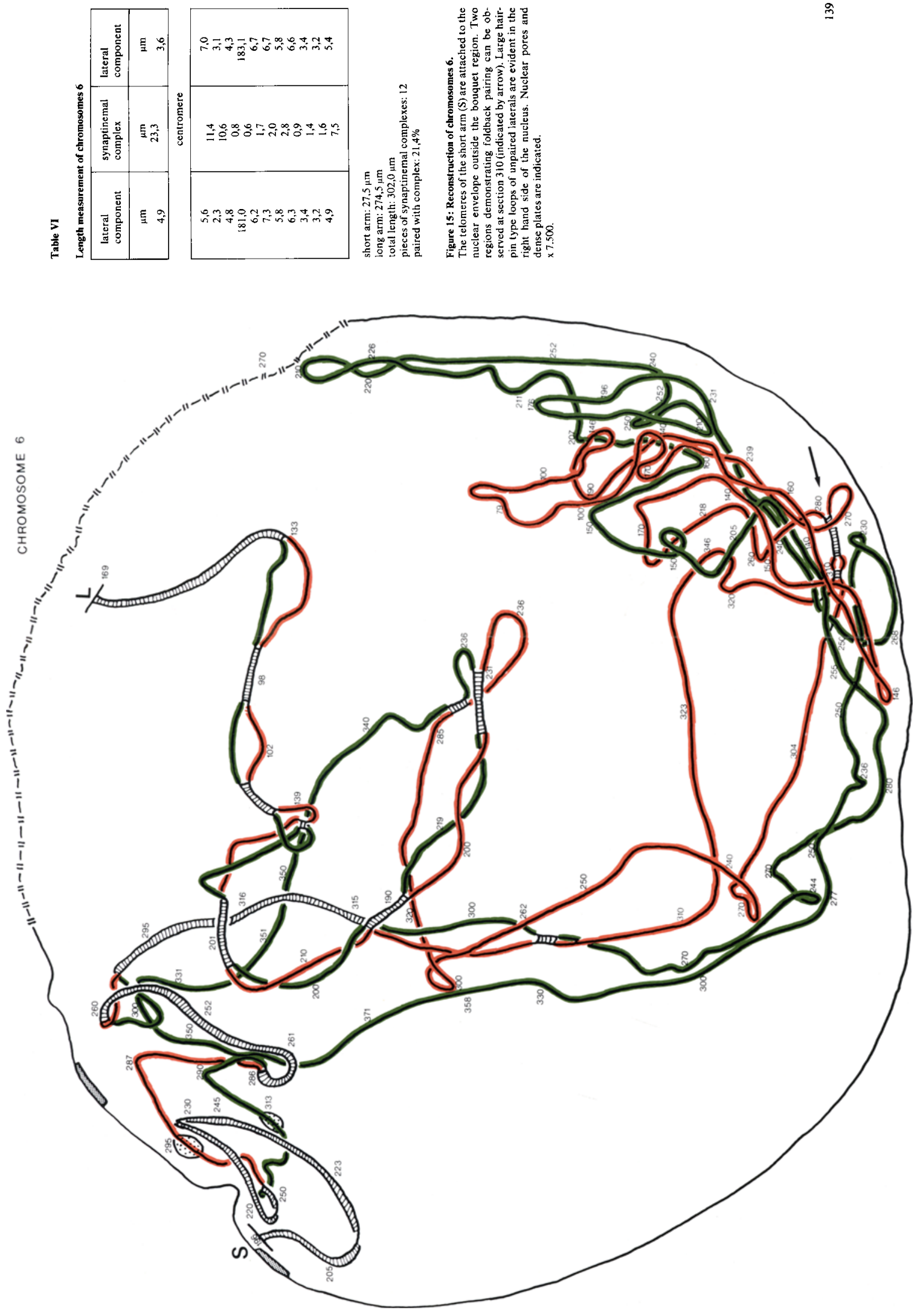

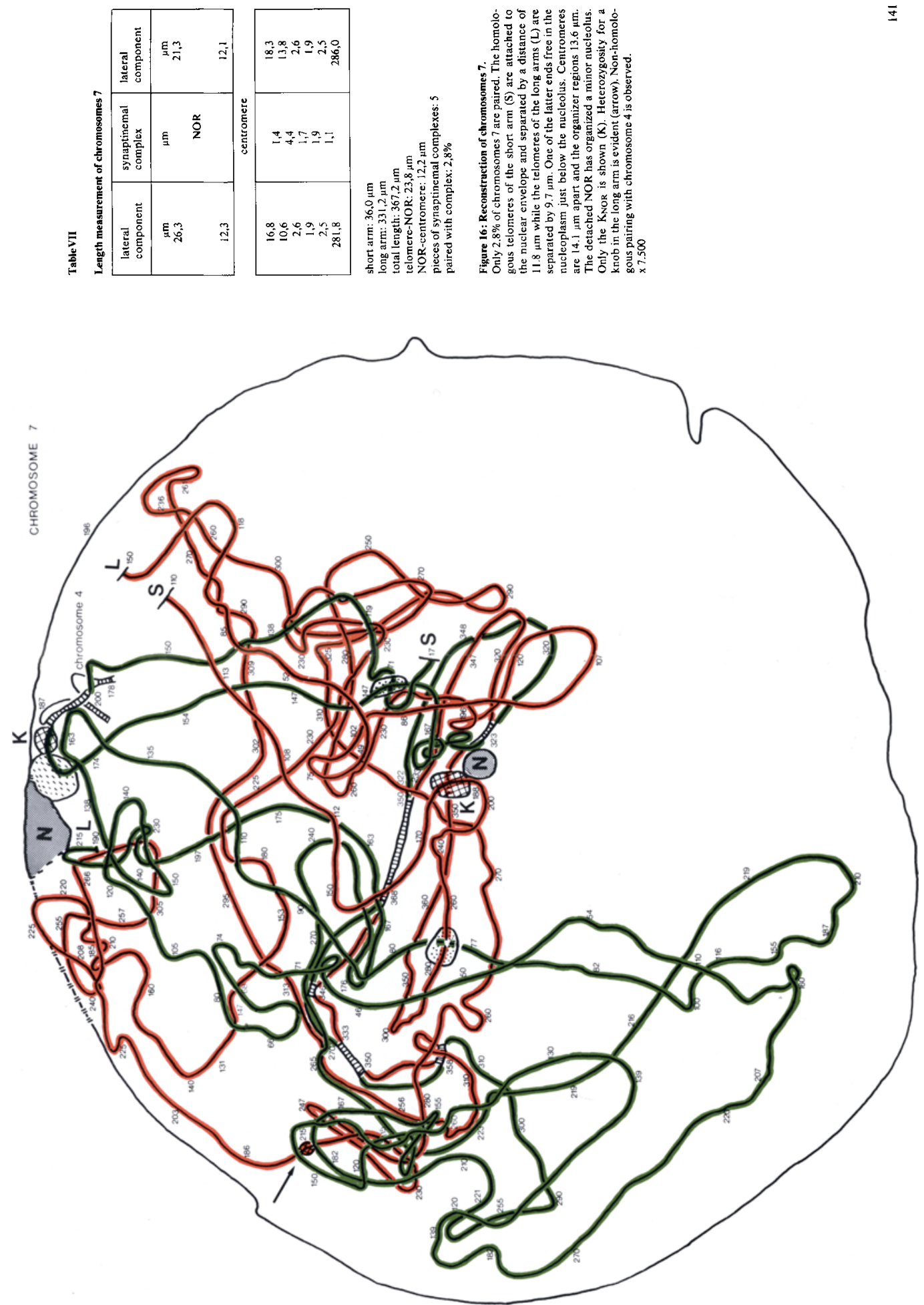

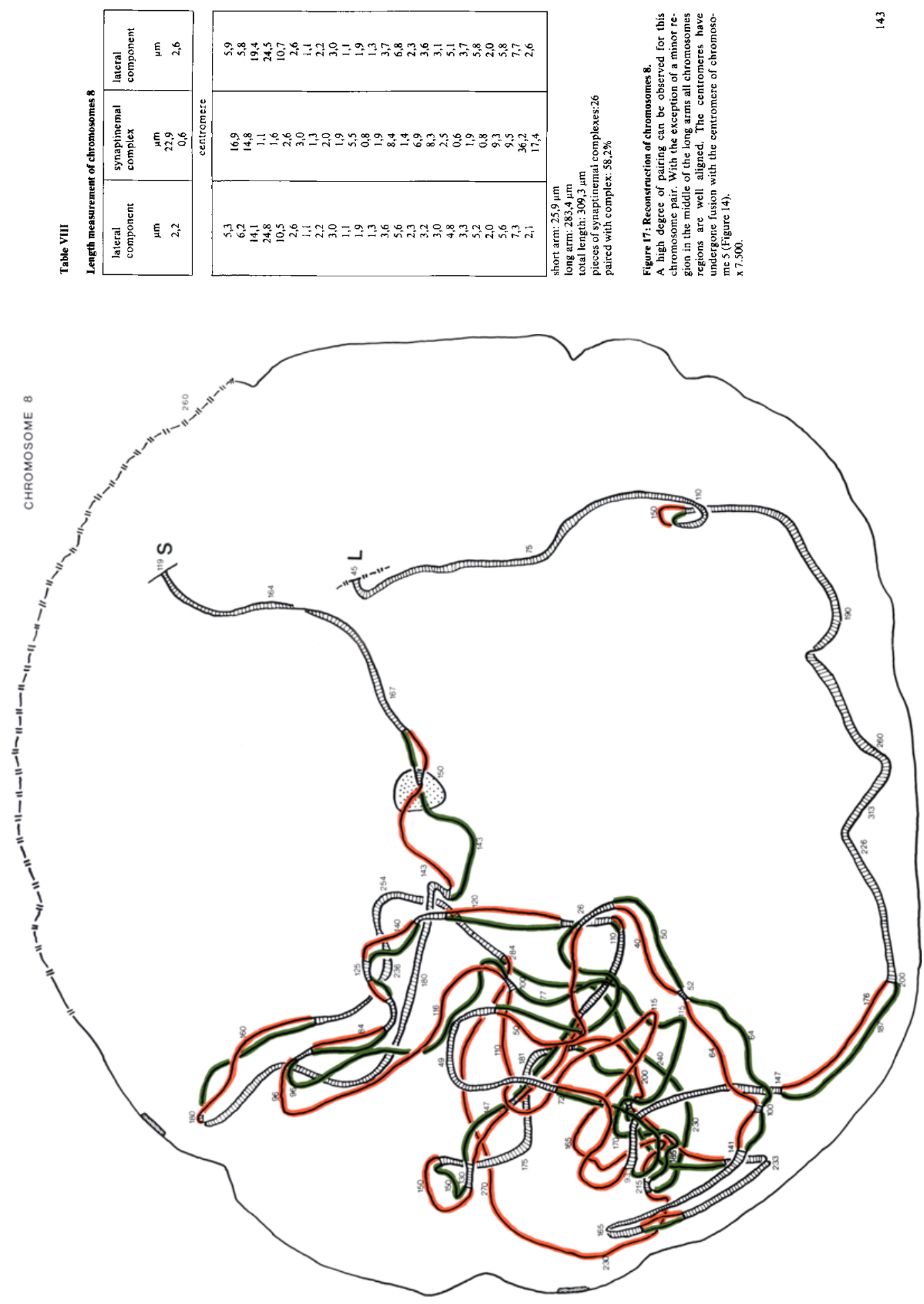

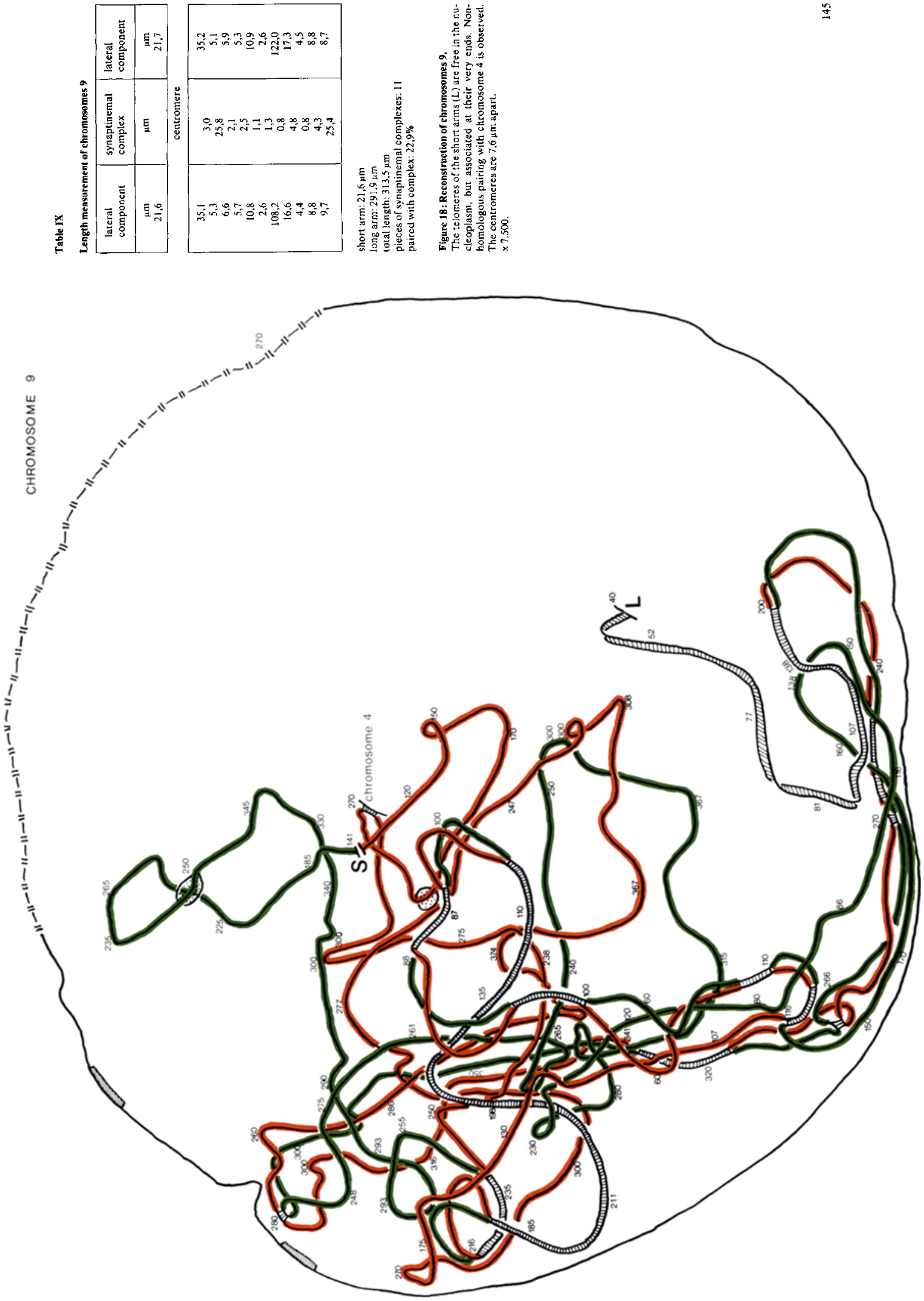

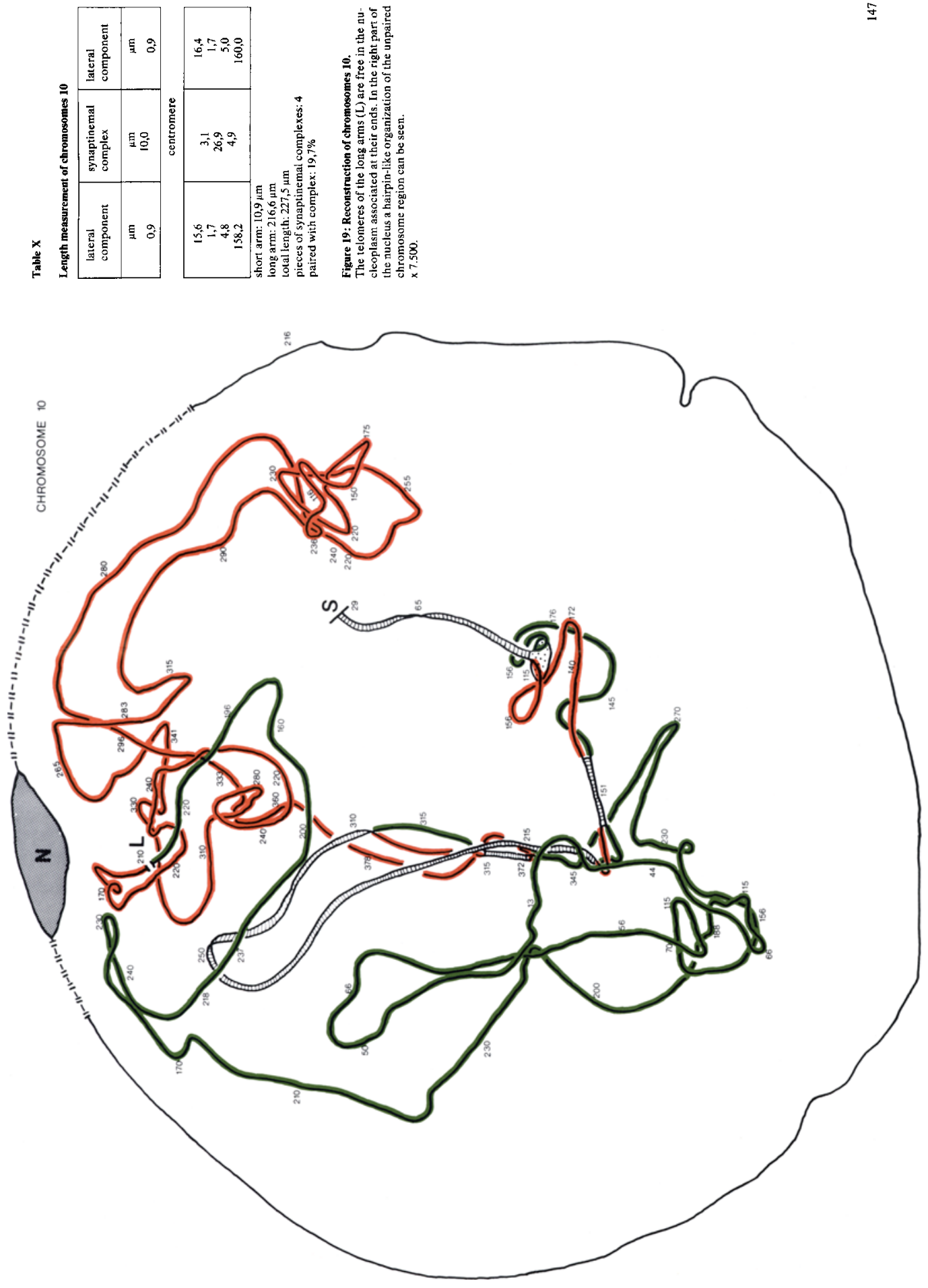

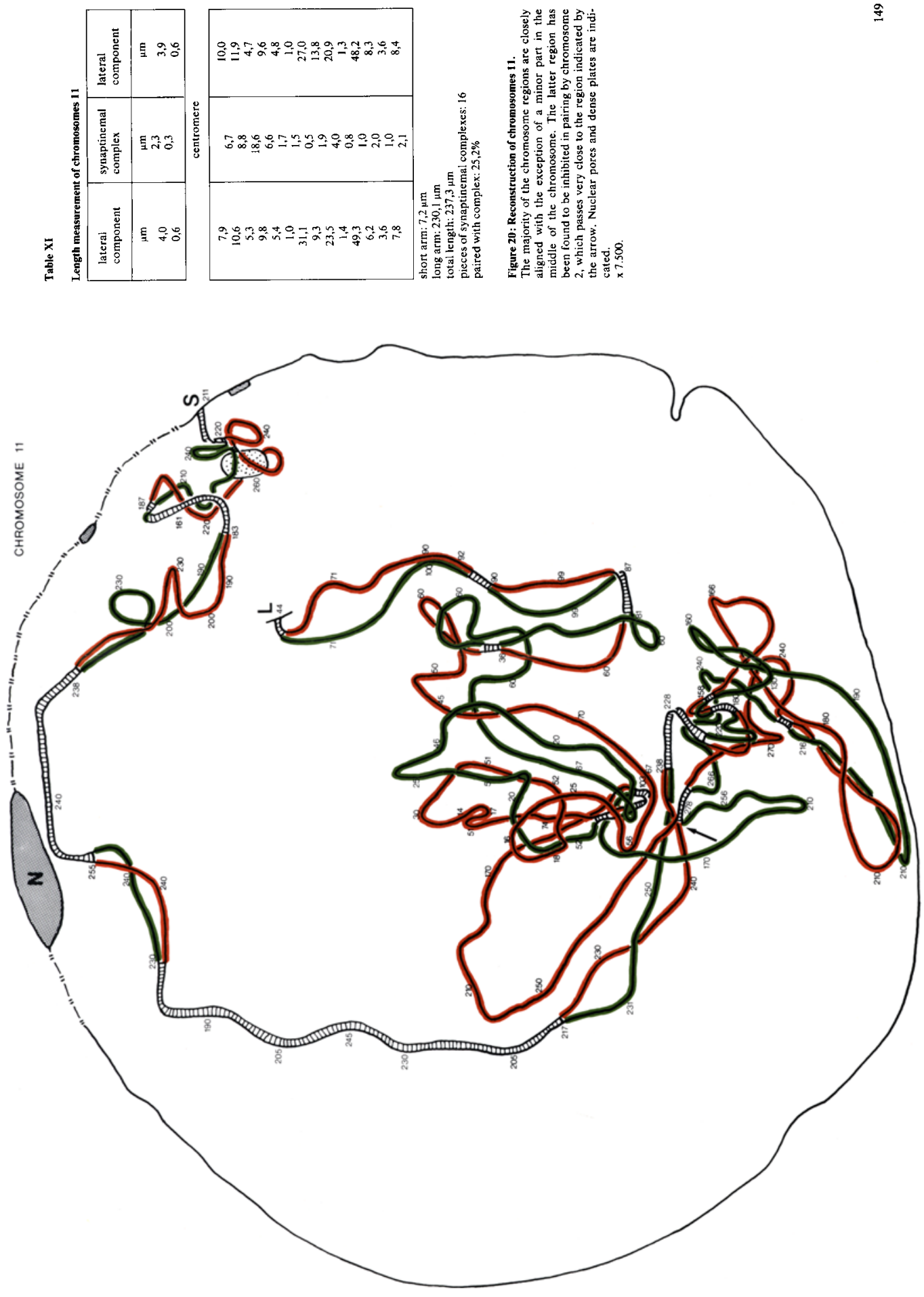

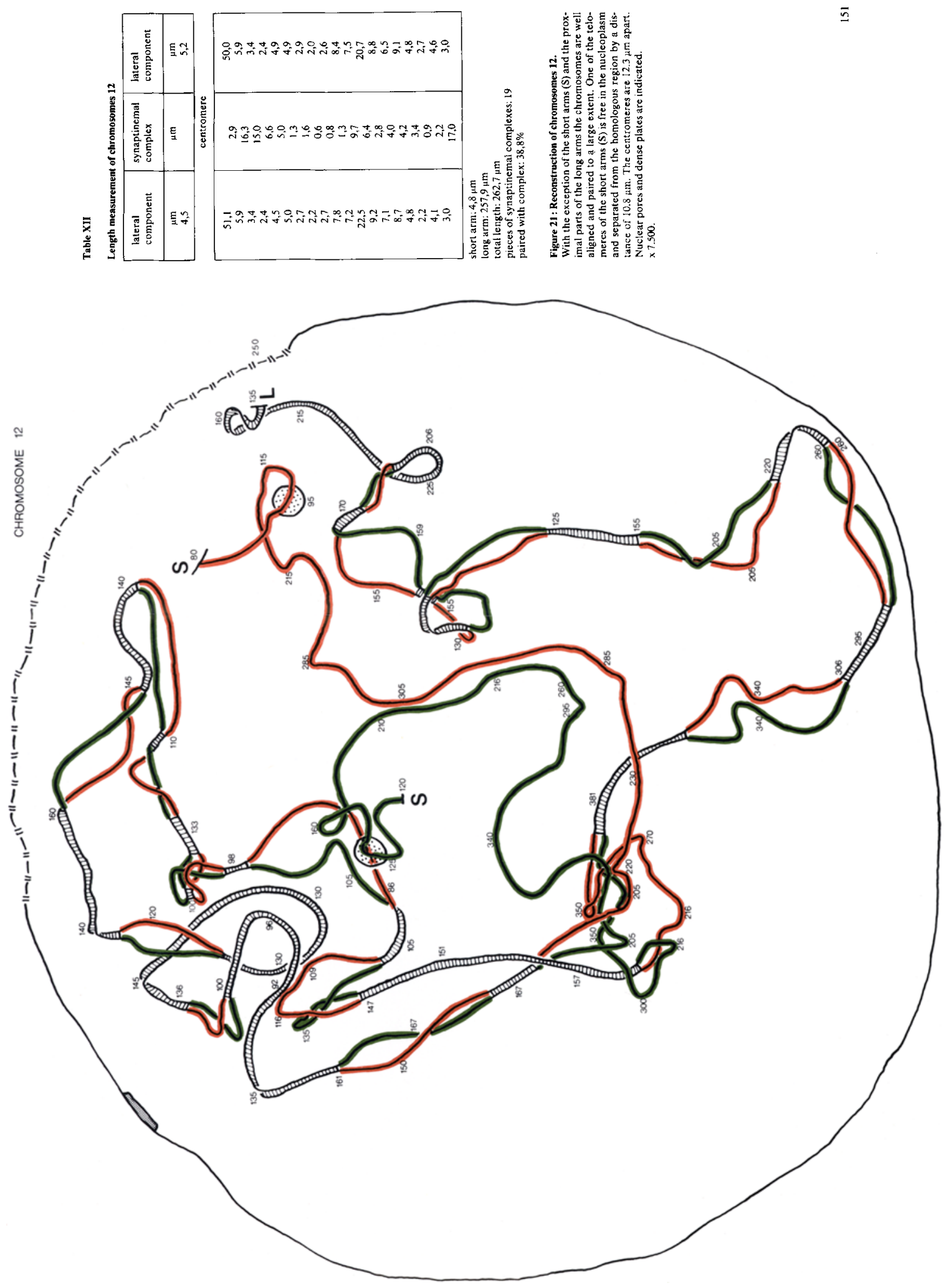